2013-01-15

\title{
Transcriptional up-regulation of ULK1 by ATF4 contributes to cancer cell survival.
}

\author{
Pike, LRG
}

http://hdl.handle.net/10026.1/10290

10.1042/BJ20120972

Biochem J

All content in PEARL is protected by copyright law. Author manuscripts are made available in accordance with publisher policies. Please cite only the published version using the details provided on the item record or document. In the absence of an open licence (e.g. Creative Commons), permissions for further reuse of content should be sought from the publisher or author. 


\title{
Transcriptional up-regulation of ULK1 by ATF4 contributes to cancer cell survival
}

\author{
Luke R. G. PIKE*, Dean C. SINGLETON, Francesca BUFFA*, Olga ABRAMCZYK†, Kanchan PHADWAL $\ddagger$, Ji-Liang LI*, \\ Anna Katharina SIMON $\neq$, James T. MURRAY $\S$ and Adrian L. HARRIS ${ }^{* 1}$ \\ *Growth Factor Group, Cancer Research UK, Molecular Oncology Laboratories, Weatherall Institute of Molecular Medicine, University of Oxford, John Radcliffe Hospital, Headington, \\ Oxford, U.K., † †entre for Cancer Research and Cell Biology, Queen's University Belfast, CCRCB Building, Belfast, U.K., †Nuffield Department of Medicine, NIHR, Biomedical Research \\ Centre, John Radcliffe Hospital, Headington, Oxford, U.K., and §School of Biochemistry and Immunology, Trinity Biomedical Sciences Institute, Romm 5.50, 152-160 Pearse Street, \\ Trinity College Dublin, Dublin 2, Ireland
}

\begin{abstract}
Hypoxia in the microenvironment of many solid tumours is an important determinant of malignant progression. The ISR (integrated stress response) protects cells from the ER (endoplasmic reticulum) stress caused by severe hypoxia. Likewise, autophagy is a mechanism by which cancer cells can evade hypoxic cell death. In the present paper we report that the autophagy-initiating kinase ULK1 (UNC51-like kinase 1) is a direct transcriptional target of ATF4 (activating transcription factor 4), which drives the expression of ULK1 mRNA and protein in severe hypoxia and ER stress. We demonstrate that ULK1 is required for autophagy
\end{abstract}

in severe hypoxia and that ablation of ULK1 causes caspase3/7-independent cell death. Furthermore, we report that ULK1 expression is associated with a poor prognosis in breast cancer. Collectively, the findings of the present study identify transcriptional up-regulation of ULK1 as a novel arm of the ISR, and suggest ULK1 as a potentially effective target for cancer therapy.

Key words: autophagy, endoplasmic reticulum stress, hypoxia, integrated stress response, UNC51-like kinase 1 (ULK1), unfolded protein response (UPR).

\section{INTRODUCTION}

The microenvironment of a solid tumour is both hostile and heterogeneous, characterized by extremes in $\mathrm{pH}$, metabolite concentrations and, in particular, partial pressure of oxygen. Rapid tumour growth leads to oxygen gradients in vivo [1,2]. Oxygen levels can be extremely low, and up to $35 \%$ of tumour cells have levels below $0.1 \% \quad \mathrm{O}_{2}$ [3]. Patients with hypoxics tumour have a significantly worse disease-free and overall survival compared with patients with well-oxygenated tumours [4,5].

Several intracellular signalling pathways known to be involved in cancer progression and adaptation are activated by hypoxia, including the well-described HIFs (hypoxia-inducible factors) $[6,7]$. In addition, in prolonged or severe hypoxia $\left(<0.01 \% \mathrm{O}_{2}\right)$, dysfunctional protein folding in the lumen of the ER (endoplasmic reticulum) leads to the activation of the UPR (unfolded protein response) [8,9]. The UPR sensors PERK [PKR (double-strandedRNA-dependent protein kinase)-like ER kinase], IRE1 (inositolrequiring enzyme 1) and ATF (activating transcription factor) 6 detect the accumulation of unfolded proteins, and elicit an adaptive response to overcome this stress [10].

The cytoplasmic kinase domain of activated PERK phosphorylates eIF $2 \alpha$ (eukaryotic initiation factor $2 \alpha$ ) and thus represses global mRNA translation [11,12], reducing the burden on the ER protein folding machinery. In addition, eIF2 $\alpha$ phosphorylation enables the selective translation of a subset of transcripts such as the key transcriptional effector ATF4 $[13,14]$. ATF4 regulates the expression of genes involved in translation, amino acid import and metabolism, redox, protein secretion, glucose metabolism, DNA damage, lipogenesis, angiogenesis, and apoptosis in what has been termed the ISR (integrated stress response) [9,15-18]. Studies have highlighted the importance of the ISR in cancer cell survival and tumour maintenance. Data indicate that ATF4 and the ISR promote the survival of hypoxia, nutrient deprivation and oxidative stress in vitro [9,19-22] and tumour progression in mouse xenograft models [19,23,24].

Cancer cells rely on autophagy to survive intratumoral hypoxia, metabolic stress and starvation, and to resist radiotherapy $[19,21,22,25]$. Intriguingly, pancreatic cancer cells and H-Rasexpressing tumour cells have elevated levels of basal autophagy, and appear to rely on autophagy to maintain high levels of oxidative phosphorylation and a pool of viable mitochondria, in a phenomenon that has been termed 'autophagy addiction' [26-28].

We and others have demonstrated that some components of the autophagic machinery are subject to ISR regulation. Severe hypoxia and ER stress induce the transcription of the essential autophagy genes $M A P 1 L C 3 B$ (microtubule-associated protein 1 light chain $3 \beta$ ) and ATG5 (autophagy-related 5) through the activity of ATF4 and CHOP [C/EBP (CCAAT/enhancerbinding protein)-homologous protein] respectively [19,21]. This up-regulation was shown to be crucial for maintaining high levels of autophagic flux in persistent hypoxia, and thus promotes cell survival. Similarly, we have demonstrated that ATF4 and autophagy are crucial for the resistance of cancer cells to bortezomib [29]. In the present study, we demonstrate that ATF4 drives the transcriptional induction of the autophagy-initiating kinase ULK1 (UNC51-like kinase 1) in response to severe hypoxia and ER stress. Furthermore, we show that ULK1 and ATF4 are crucial for autophagy, mitophagy and cancer cell survival in both normoxia and severe hypoxia, and that loss of

Abbreviations used: ACC, acetyl-CoA carboxylase; AMPK, AMP-activated protein kinase; ATF, activating transcription factor; ATG5, autophagy-related 5; AV, annexin V; CAIX, carbonic anhydrase IX; ChIP, chromatin immunoprecipitation; CHOP, C/EBP (CCAAT/enhancer-binding protein)-homologous protein; CREB, CAMP-response-element-binding protein; elF2 $\alpha$, eukaryotic initiation factor $2 \alpha$; ER, endoplasmic reticulum; HMGB1, high-mobility group box 1; ISR, integrated stress response; LC3, microtubule-associated protein light chain 3; MAP1LC3B, microtubule-associated protein 1 light chain 3 $\beta$; MBP, myelin basic protein; mTOR, mammalian target of rapamycin; P-, phosphorylated; PERK, PKR (double-stranded-RNA-dependent protein kinase)-like ER kinase; Raptor, regulatory associated protein of mTOR; RNAi, RNA interference; RT-qPCR, real-time quantitative PCR; shRNA, short hairpin RNA; siRNA, small interfering RNA; S6K, S6 kinase; TG, thapsigargin; TN, tunicamycin; ULK1, UNC51-like kinase 1; UPR, unfolded protein response.

To whom correspondence should be addressed (email aharris.lab@imm.ox.ac.uk). 
ULK1 promotes necrotic cell death. Finally, we show that elevated ULK1 mRNA expression is associated with poor prognosis in a specific group of breast cancer patients.

\section{MATERIALS AND METHODS}

\section{Reagents and cell culture}

TG (thapsigargin) (catalogue number 586005), TN (tunicamycin) (catalogue number 654380), MG115 (catalogue number 474780), bafilomycin A1 (catalogue number 196000) and pepstatin A (catalogue number 516481) were obtained from Calbiochem, and E64D (catalogue number E8640) and chloroquine (catalogue number C6628) were obtained from Sigma-Aldrich. Bortezomib was a gift from Millennium Pharmaceuticals. A431, MCF7, HT29, MDA-MB-231, HCT116 and U87 cell lines were provided by Cancer Research UK and were cultured in DMEM (Dulbecco's modified Eagle's medium; Invitrogen, catalogue number 11965) with $10 \%$ (v/v) FBS (fetal bovine serum), supplemented with $0.5 \mu \mathrm{g} / \mathrm{ml}$ penicillin/streptomycin (Invitrogen, catlogue number 15070) and $4 \mathrm{mM}$ L-glutamine (Invitrogen, catalogue number 25030). Cell lines were incubated at $37^{\circ} \mathrm{C}$ in a non-humidified incubator with $95 \%$ air supplemented with $5 \% \mathrm{CO}_{2}$.

\section{Hypoxic incubations}

For incubations in severe hypoxia $\left(<0.01 \% \mathrm{O}_{2}\right)$, the $\mathrm{InVivO}_{2}$ 400 humidified gas-sorted incubator with a glove box (Ruskin) was used. A gas mix of $5 \% \mathrm{H}_{2}, 5 \% \mathrm{CO}_{2}$ and $90 \% \mathrm{~N}_{2}$ was fed into the system. A palladium catalyst maintained near-zero $\mathrm{O}_{2}$ concentrations by reducing trace $\mathrm{O}_{2}$. For incubations in moderate hypoxia $\left(0.1 \% \mathrm{O}_{2}\right)$, a Heto-Holten CellHouse 170 incubator (RS Biotech) was used.

\section{Transfections}

Transfections of siRNA (small interfering RNA) and DNA were performed with HiPerfect (Qiagen, catalogue number 301704) and FuGENE $^{\circledR} 6$ (Roche, catalogue number 11814443001) transfection reagents respectively, as described previously [29]. The specific siRNA sequences used can be found in Supplementary Table S1 (at http://www.biochemj.org/bj/449/bj4490389add. htm).

\section{Western blot analysis}

Western blots were prepared as described previously [29]. Antibodies used are described in the Supplementary Online data (at http://www.biochemj.org/bj/449/bj4490389add.htm).

\section{RT-qPCR (real-time quantitative PCR)}

In RT-qPCR experiments, the methods used by us for extraction, quantification and evaluation of the quality of RNA were as described previously [30]. Data from RT-qPCR experiments were normalized to tubulin $\alpha 6$. The sequences of primers used are listed in Supplementary Table S2 (at http://www.biochemj. org/bj/449/bj4490389add.htm).

\section{Immunocytochemistry for LC3 (microtubule-associated protein light chain 3)}

Staining for LC3-positive foci was performed as described previously [29].

\section{ULK1 activity assays}

Endogenous ULK1 protein was immunoprecipitated from A431 and MCF7 cells and used in subsequent analysis of MBP (myelin basic protein) phosphorylation. A detailed method is given in the Supplementary Online Data.

\section{ChIP (chromatin immunoprecipitation)}

The ChIP assays were performed using the EZ ChIP Chromatin Immunoprecipitation kit (Millipore, catalogue number 17-371), according to the manufacturer's instructions, using $5 \mu \mathrm{g}$ of rabbit anti-ATF4 antibody. The sequences of primers used for RT-qPCR of promoter fragments are shown in Supplementary Table S3 (at http://www.biochemj.org/bj/449/bj4490389add.htm).

\section{Imagestream ${ }^{\circledR}$ analysis of autophagy}

MCF7 cells were stained with a live/dead marker, Lysotracker Red, and anti-LC3 antibodies and fixed and analysed for autophagic flux using Imagestream ${ }^{\circledR}$ flow-cytometric methods as described previously [31].

\section{Flow cytometry}

MCF7 and A431 cells were stained with Alexa Fluor ${ }^{\circledR} 647$ conjugated anti-AV (annexin-V) antibody (Invitrogen, catalogue number A23204) and cell death was quantified by flow cytometry according to the manufacturer's protocol and as decribed previously [32] using a Cyan ADP Flow Cytometer (Dako). Mitochondrial fluorescence was measured by first staining cells with Mitotracker Red FM (Invitrogen, catalogue number M22425). Mean fluorescence was used to assess changes in mitochondrial mass, as described previously [33].

\section{Clonogenic assays}

Clonogenic assays were performed to assess survival in severe hypoxia following transfection with siRNA molecules specific for ATF4 or ULK1, as described previously [21]. For the counting of colonies, the ColCount Colony Counter (Oxford Optronix) was used.

\section{Luminescent assays}

Caspase 3/7 protease activity was analysed using the CaspaseGlow $^{\circledR}$ 3/7 assay (Promega, catalogue number G8091) according to the manufacturer's protocol. Similarly, ATP levels were measured using the Cell-Titer Glo ${ }^{\circledR}$ Luminescent Cell Viability assay (Promega, catalogue number G7570) according to the manufacturer's protocol.

\section{Immunohistochemistry}

Immunohistochemistry was performed on spheroids to assess clinically relevant markers of hypoxia, cell death and proliferation as described previously [29].

\section{Three-dimensional growth assay}

Rapid generation of single-tumour spheroids was achieved using the method developed by Ivascu and Kubbies [34]. An Axiovert 135 Microscope (Zeiss) was used for image capture and ImageJ version $1.43(\mathrm{NIH})$ was used to calculate spheroid volume. 


\section{HMGB1 (high-mobility group box 1) ELISA}

An HMGB1 sandwich ELISA from IBL International was used to quantitatively determine the levels of HMGB1 in the cell culture medium of cells undergoing necrosis, according to the manufacturer's protocol.

\section{Survival analysis}

For survival analysis, a published series of breast cancer patients was used. mRNA was extracted from tumour samples, and expression profiling was performed as described previously [35]. Full details are given in the Supplementary Online Data. Written informed consent was obtained and all clinical investigations were conducted according to the ethical standards and principles expressed in the Declaration of Helsinki. Ethical approval was obtained from the local research ethics committee.

\section{RESULTS}

ULK1 mRNA and protein levels increase in response to severe hypoxia, ER stress, moderate hypoxia and anti-angiogenic therapy

In previous work, we identified hypoxia- and ATF4-dependent genes by microarray analysis [21]. The top 25 of these genes were validated by RT-qPCR analysis and published [21]. Further analysis of our data revealed that $U L K 1 \mathrm{mRNA}$ was increased 1.76 -fold by severe hypoxia $(P=0.001)$ and then reduced to 0.60 fold after knockdown of ATF4 $(P=0.004)$.

To confirm the microarray results identifying ULK1 as a hypoxia-inducible gene, a panel of cell lines were exposed to severe hypoxia for increasing periods of time and ULK1 mRNA levels were assessed by RT-qPCR. In A431, HT29 and MCF7 cells, ULK1 mRNA was seen to increase after $12 \mathrm{~h}$ in severe hypoxia, and to reach a maximum after $48 \mathrm{~h}$ (Figures 1A-1C). A431 and HT29 cells exhibited close to 30-fold increases in $U L K 1 \mathrm{mRNA}(P<0.05$ and $P<0.01$ respectively), whereas MCF7 cells exhibited a more modest increase of 5-fold $(P<0.01)$.

Consistent with the mRNA data, ULK1 protein levels in A431 and HT29 cells increased markedly after 12 and $6 \mathrm{~h}$ in severe hypoxia respectively (Figures $1 \mathrm{D}$ and $1 \mathrm{E}$ ). In contrast, in MCF7 cells ULK1 protein levels were found to be high in normoxia, and then decreased after $48 \mathrm{~h}$ in severe hypoxia (Figure 1F). In U87 and HCT116 cells, ULK1 protein peaked at $6 \mathrm{~h}$ and then rapidly decreased with prolonged exposure to severe hypoxia (Supplementary Figures S1A and S1B at http://www.biochemj.org/bj/449/bj4490389add.htm).

Maximal induction of ULK1 mRNA and protein was preceded by the accumulation of ATF4 protein (Figures 1A-1F). As reported previously [19,21], LC3-II protein levels increased markedly after $12-24 \mathrm{~h}$ in severe hypoxia, indicating that autophagy was increased in this condition. Likewise, the expression of the ISR target gene CHOP showed an mRNA and protein expression profile that mimicked that of ULK1 (Supplementary Figures S1C-S1E).

To confirm that LC3-II protein levels were indicative of increased autophagic flux rather than decreased lysosomal degradation, p62 protein levels were examined. Indeed, severe hypoxia resulted in increased degradation of $\mathrm{p} 62$ protein in concert with LC3-II protein induction, whereas LC3-I remained constant throughout (Figures $1 \mathrm{G}$ and $1 \mathrm{H}$ ).

Similarly, when A431 cells were exposed to TG or TN, potent stimulators of the ISR and autophagy [36], ATF4 protein accumulated after $2 \mathrm{~h}$ and was followed by ULK1 protein expression after 24 or $6 \mathrm{~h}$ respectively (Figures $1 \mathrm{I}$ and $1 \mathrm{~J}$ ). When A431 cells were exposed to moderate hypoxia $\left(0.1 \% \mathrm{O}_{2}\right)$, a similar pattern of induction was observed (Figure 1K), indicating that this phenotype is not limited to severe hypoxia.

To assess whether ULK1 may be induced by intratumoral hypoxia, mRNA was isolated from U87 tumour xenografts treated with PBS or bevacizumab [humanized anti-VEGF (vascular endothelial growth factor) antibody], a treatment well known to reduce intratumoral oxygen levels [37,38]. ULK1 mRNA levels were increased nearly 2 -fold by bevacizumab treatment $(P<0.001)$, in a fashion similar to CHOP (Supplementary Figure S2A at http://www.biochemj.org/bj/449/bj4490389add.htm). Similarly, when A431 cells were grown as spheroids in vitro, ULK1 protein was found to accumulate after 3 days, and to reach a maximum after 6 days growth, correlating with expression of ATF4 and LC3-II (Supplementary Figure S2B). These spheroids were confirmed to be hypoxic by staining for pimonidazole, CAIX (carbonic anhydrase IX) and CHOP (Supplementary Figure S2C).

As mentioned above, MCF7 cells exposed to severe hypoxia strongly induced ULK1 mRNA, although ULK1 protein levels were seen to decrease. Interestingly, proteasome inhibition with bortezomib or MG115 partially blocked the reduction in ULK1 caused by prolonged exposure to severe hypoxia (Supplementary Figure S3A at http://www.biochemj.org/bj/449/ bj4490389add.htm), whereas inhibitors of the lysosome or caspases failed to rescue ULK1 expression (Supplementary Figures S3B and S3C).

\section{Severe hypoxia and ER stress induce ULK1 in an ATF4-dependent manner}

Our microarray analysis identified ULK1 as a transcriptional target of ATF4 [21]. To confirm this result, A431 and MCF7 cells were treated with siRNA to deplete ATF4. In both cell lines, this treatment reduced ATF4 protein by at least $50 \%$ after exposure to severe hypoxia (Figures $2 \mathrm{~A}$ and 2C). ATF4 knockdown reduced CHOP protein (Figures $2 \mathrm{~A}$ and $2 \mathrm{C}$ ) and mRNA induction (Supplementary Figures S4A and S4B at http://www.biochemj.org/bj/449/bj4490389add.htm), indicating that the transcriptional activity of ATF4 was attenuated. Similarly, ULK1 protein expression in both A431 and MCF7 cells was abrogated in both severe hypoxia and normoxia (Figures 2A and 2C), as was ULK1 mRNA induction (Figures 2B and 2D) $(P<0.05$ and $P<0.01$ for A431 and MCF7 respectively). Likewise, the accumulation of ULK1 protein caused by TG or TN treatement was completely blocked when A431 cells were first depleted of ATF4 by RNAi (RNA interference) (Figure 2E). Interestingly, however, ectopic expression of ATF4 in normoxia was not sufficient to induce the expression of ULK1 in A431 cells to the levels seen in severe hypoxia (Supplementary Figure S5 at http://www.biochemj.org/bj/449/bj4490389add.htm).

\section{ATF4 binds directly to the promoter region of ULK1}

Using the Genomatix ${ }^{\circledR}$ online software suite, we were able to identify several putative CREB (cAMP-response-elementbinding protein)/ATF-binding sites (containing the TGACG core sequence) in the $U L K 1$ promoter. Thus, to determine whether ATF4 binds directly to the promoter region of $U L K 1$, ChIP was performed using antibodies specific for ATF4.

Using RT-qPCR primers spanning the region $2000 \mathrm{bp}$ upstream of the transcription start site, the first exon and $500 \mathrm{bp}$ downstream of translation start (Figure 2F), we observed that ATF4 ChIP samples showed the greatest enrichment in fragments 
A

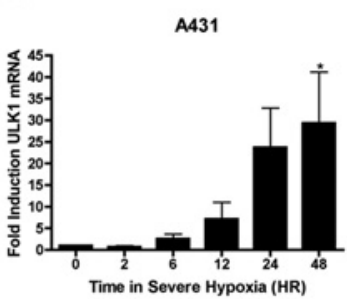

D A431 Time in Severe Hypoxia(HR) \begin{tabular}{lllllll}
\hline 0 & 2 & 6 & 12 & 24 & 48
\end{tabular} ULK1 $----\pi=$ ATF4 $=-$ $\mathrm{CHOP}$ LC3-II Actin

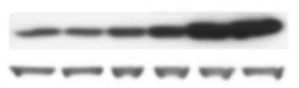

G A431 Time in Severe Hypoxia(HR) \begin{tabular}{lllll}
\hline 0 & 6 & 12 & 24 & 48
\end{tabular}
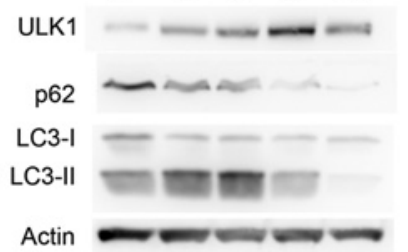

I A431 \begin{tabular}{llllll} 
TG Treatment (HR) \\
\hline 0 & 2 & 6 & 12 & 24 & 48
\end{tabular}

ULK1

ATF4 CHOP LC3-II Actin

J A431
B

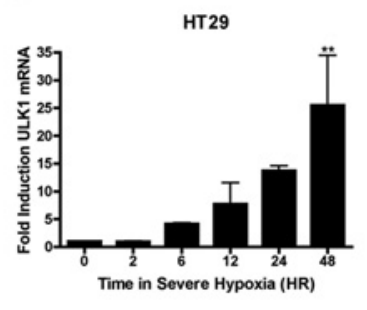

E HT29

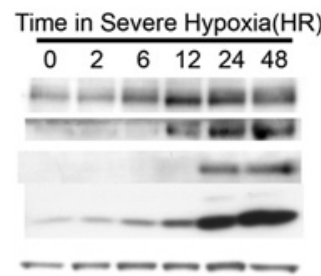

H MCF7
C

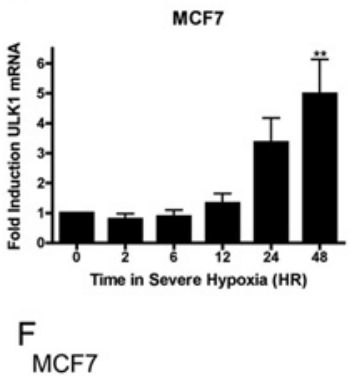

Time in Severe Hypoxia(HR)

$$
\begin{array}{llllll}
\hline 0 & 2 & 6 & 12 & 24 & 48
\end{array}
$$

$=-m=$
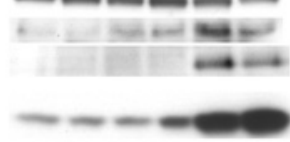

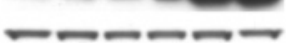

Time in Severe Hypoxia(HR)

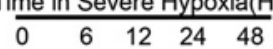

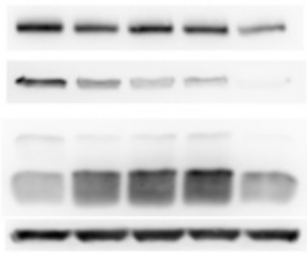

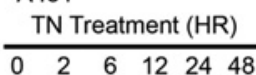

K A431

Time in Moderate Hypoxia(HR)
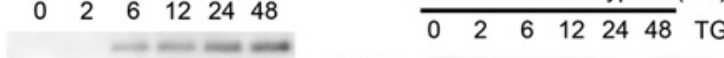

ULK1

ATF4

CHOP

$H I F 1 \alpha$

CAIX

LC3-II

Actin

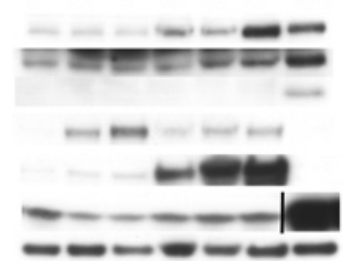

\section{Figure 1 ULK1 is induced by ER stress and hypoxia in vivo and in vitro}

A431, HT29 and MCF7 cells were exposed to severe hypoxia $\left(<0.01 \% 0_{2}\right)$ for the times indicated, after which time cells were harvested and analysed by RT-qPCR $(\mathbf{A}-\mathbf{C})$ or Western blotting $(\mathbf{D}-\mathbf{H})$ using the primers and antibodies indicated. A431 cells were treated with $300 \mathrm{nM} \mathrm{TG}(\mathbf{I}), 5 \mu \mathrm{g} / \mathrm{ml}$ TN $(\mathbf{J})$ or exposed to moderate hypoxia $\left(0.1 \% \mathrm{O}_{2}\right)(\mathbf{K})$ and similarly analysed. The vertical line in (K) indicates the point where the LC3 blot was cut. ${ }^{*} P<0.05$ and ${ }^{\star \star} P<0.01$ respectively.

corresponding to the CREB site spanning $-306 \mathrm{bp}$ to $-285 \mathrm{bp}$ relative to the ATG of exon one (Figure $2 \mathrm{G}$ ). Furthermore, the signal obtained from this primer set increased with exposure to severe hypoxia, reflecting increased ATF4 binding with severe hypoxia (Figure $2 \mathrm{H}$ ). A previously published set of primers designed against the $C H O P$ promoter was used as a positive control [19].

\section{Severe hypoxia increases cellular ULK1 kinase activity through both transcriptional and post-translational mechanisms}

To date, most work has focused on post-translational regulation of the specific activity of ULK1, demonstrating an important role for AMPK (AMP-activated protein kinase)- and mTOR (mammalian target of rapamycin)-mediated phosphorylation of ULK1 in fasting and fed states respectively [39-43], rather than the transcriptional regulation of total ULK1 levels. Thus, having found that $U L K 1 \mathrm{mRNA}$ up-regulation by ATF4 results in increased protein expression in A431 cells but not in MCF7 cells, we next sought to assess the total and specific activity of ULK1 in each case.

The total immunoprecipitated ULK1 activity total P-MBP (Pindicates phosphorylated protein)] from A431 cells increased by $103 \%$ after $24 \mathrm{~h}$ in severe hypoxia (Figure 3A), although, interestingly, the specific ULK1 activity (normalized to protein expression) was $35 \%$ lower (Figure 3B). Thus, in spite of decreased enzymatic activity, the ATF4-mediated increase in ULK1 protein led to increased ULK1 activity and autophagy in severe hypoxia. In MCF7 cells, on the other hand, although ULK1 

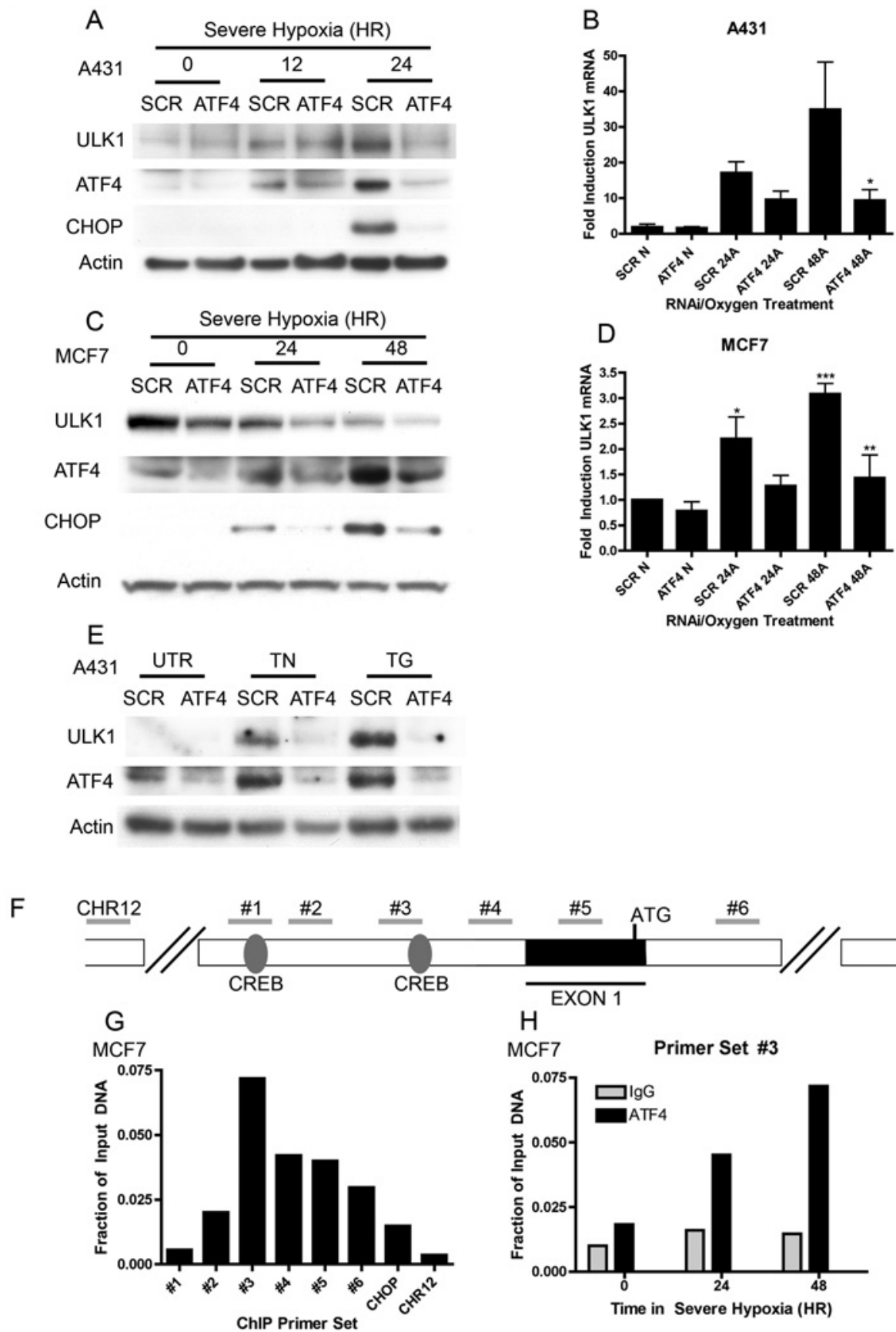

Figure 2 ATF4 binds directly to the ULK1 promoter to drive ULK1 expression in severe hypoxia or due to ER stress

A431 (A and B) or MCF7 (C and D) cells were transfected with siRNA against ATF4 (ATF4) or a scrambled control sequence (SCR) overnight and exposed to severe hypoxia (A) or normoxia (N) the following day. After 12,24 or $48 \mathrm{~h}$, cells were harvested for analysis by Western blotting or RT-qPCR using the antibodies and primers indicated. ${ }^{\star} P<0.05$, ${ }^{\star \star} P<0.01$ and ${ }^{\star \star \star} P<0.001$ respectively. (E) A431 cells were similarly transfected and then untreated (UTR) or treated with $300 \mathrm{nM} \mathrm{TG}$ or $5 \mu \mathrm{g} / \mathrm{ml} \mathrm{TN}$ for $24 \mathrm{~h}$ before harvesting and analysis by Western blotting. MCF7 cells were exposed to severe hypoxia and then processed for ATF4 ChIP. Primers sets (represented by grey bars) were designed spanning the region 2000 bp upstream of the transcription start site, the first exon and 500 bp downstream of the translation start containing two putative ATF/CREB-binding sites. (F) Locations of primer pairs used for RT-qPCR analysis following ChIP. ATG indicates the translation start site in exon 1. // indicates large gaps upstream and downstream of the ULK1 gene. CHR12 indicates primers designed against an upstream region on chromosome 12

(G) RT-qPCR was performed to analyse the enrichment of these ULK1 promoter fragments in the hypoxic samples. CHOP indicates a previously published primer set for the CHOP promoter [19]. (H) RT-qPCR against the region corresponding to primer set \#3 showed hypoxia-dependent enrichment of the ATF4-pulldown samples relative to the IgG controls. Data shown are from a single ChIP, in which three independent dishes were pooled for each time point. Two such independent experiments were performed.

protein did not accumulate in severe hypoxia, its specific activity increased by $115 \%$ after $12 \mathrm{~h}$ in severe hypoxia $(P<0.05)$ (Figure 3B), yielding an overall increase in total ULK1 activity of nearly $81 \%$ at this time point $(P<0.05)$ (Figure $3 \mathrm{~A})$. However, this increase in ULK1 activity was transient and, by $24 \mathrm{~h}$ in severe hypoxia, both specific and absolute ULK1 activity had decreased to normoxic levels (Figures $3 \mathrm{~A}$ and $3 \mathrm{~B}$ ).

These changes in the activity of ULK1 were associated with increased AMPK signalling, as reflected by the increased level of P-AMPK and its products P-ACC (acetyl-CoA carboxylase) and 


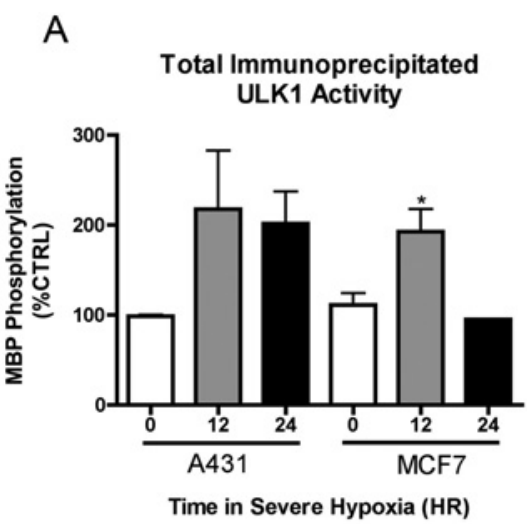

C
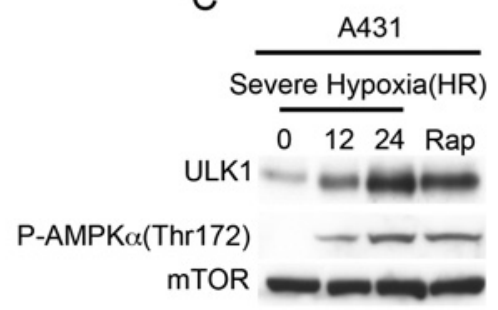

P-p70 S6K(Ser371)

p70 S6K

P-Raptor(Ser792)

Raptor

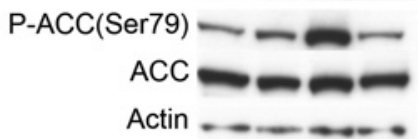

B

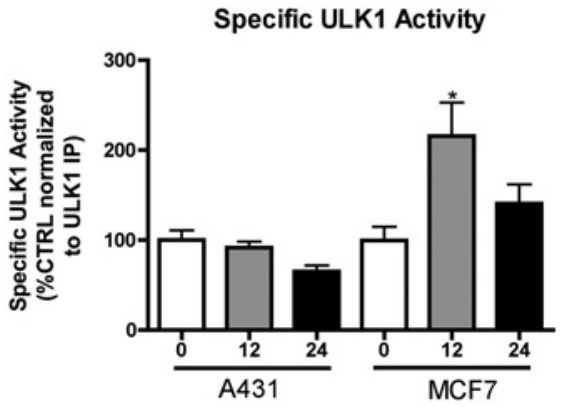

Time in Severe Hypoxia (HR)

$\mathrm{D}$

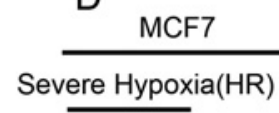

$0 \quad 1224$ Rap

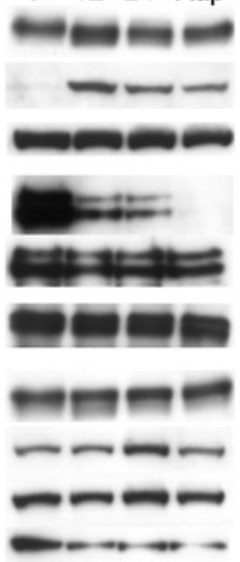

$\mathrm{E}$

A431

Total Immunoprecipitated ULK1 Activity

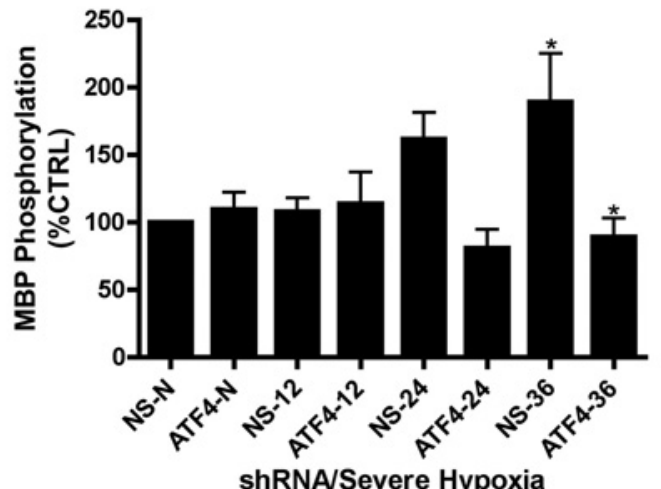

Figure 3 ULK1 activity increases with severe hypoxia and is dependent on ATF4

A431 or MCF7 cells were exposed to severe hypoxia for 12 or $24 \mathrm{~h}$, or with $100 \mathrm{nM}$ rapamycin for $24 \mathrm{~h}$, after which time cells were harvested and lysates prepared. An ULK1-specific antibody was used to immunoprecipitate endogenous ULK1, and an in vitro ULK1 kinase activity was performed using MBP as a substrate. (A) Total immunoprecipitated ULK1 activity was calculated as MBP phosphorylation normalized to the control. (B) Specific ULK1 activity was calculated as the ratio of MBP phosphorylation to ULK1 protein (densitometry), normalized to the control. (C and D) Alternatively, lysates were subjected to analysis by Western blotting, using the antibodies indicated. (E) A431 cells stably expressing an inducible shRNA construct for ATF4 (ATF4) or a non-silencing control (NS) were treated with doxycyclin for 2 days and then exposed to severe hypoxia for the times indicated. Total immunoprecipitated ULK1 activity was similarly quantified.

P-Raptor (regulatory associated protein of mTOR), and decreased mTOR signalling, as reflected by decreased P-p70S6K (S6 kinase) (Figures 3C and 3D). Strikingly, severe hypoxia also caused a downward shift of the ULK1 band, which may be indicative of hyper-dephosphorylation, as observed previously in starvation [39]. 

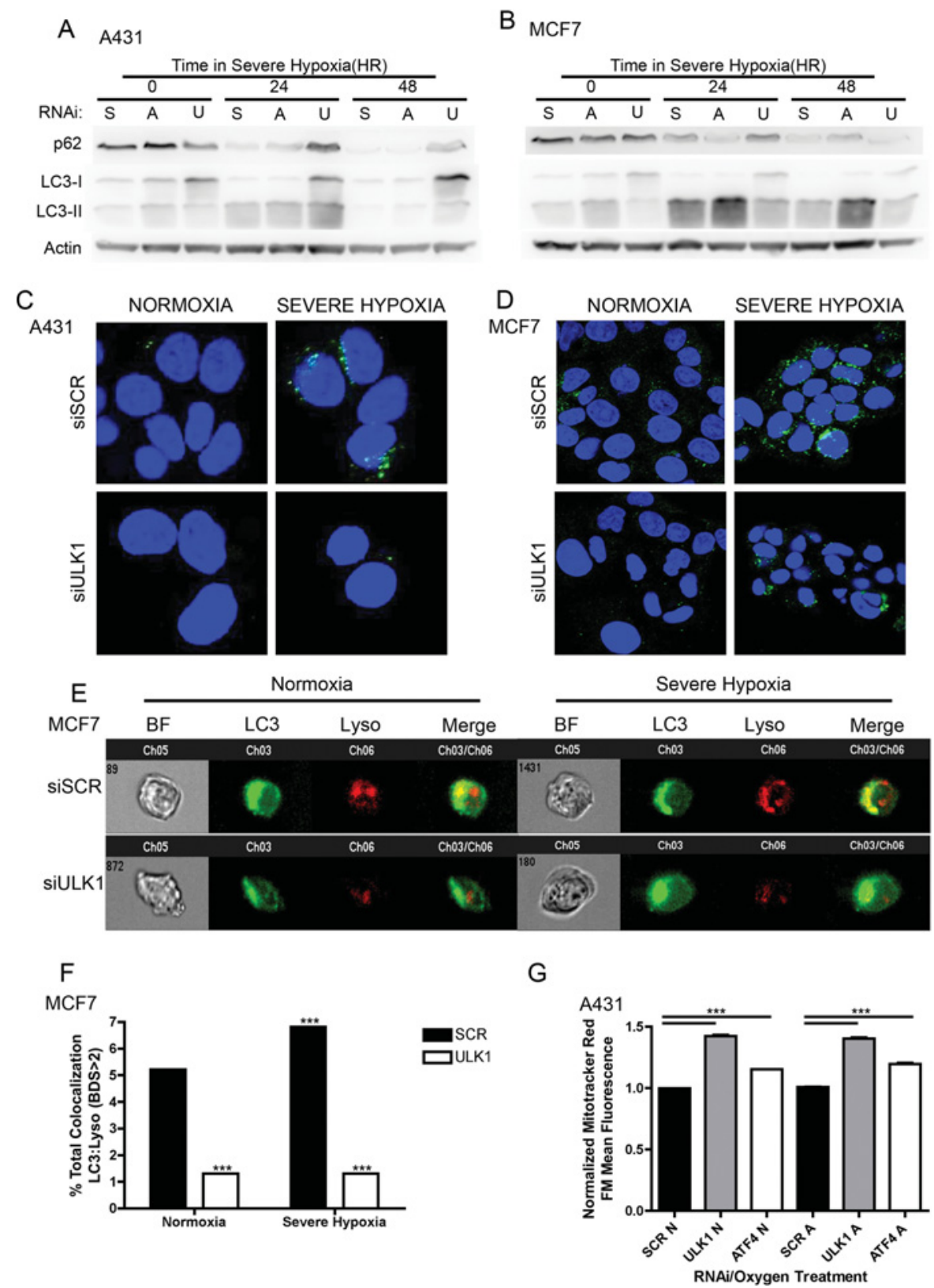

\section{Figure 4 ULK1 is required for hypoxia-induced autophagy}

A431 (A and C) or MCF7 (B and D) cells were transfected with siRNA against ULK1 (U), ATF4 (A) or a scrambled control sequence (S) overnight and exposed to severe hypoxia the next day for the times indicated. (A and B) Cells were harvested for analysis by Western blotting using the antibodies indicated. (C and D) Cells were fixed and stained for LC3 (green) and counterstained with DAPI (4',6-diamidino-2-phenylindole) (blue). (E) MCF7 cells were transfected with siRNA against ULK1 or a scrambled control sequence (SCR) overnight and exposed to severe hypoxia the next day. At $24 \mathrm{~h}$ later, cells were stained for LC3 (green) and lysosomes (red), and fixed for Imagestream ${ }^{\circledR}$ analysis. (F) Histogram indicating co-localization of LC3 with lysosomes with bright detail similarity $(B D S)>2$. (G) Alternatively, Mitotracker Red FM dye was added to live cells and cells were analysed by flow cytometry. Mean fluorescence was normalized to the normoxic control. N, normoxia; A, severe hypoxia. ${ }^{\star \star *} P<0.001$.

To confirm the role of ATF4 in the transcriptional regulation of ULK1 activity, A431 cells expressing an inducible lentiviral shRNA (short hairpin RNA) sequence against ATF4 were treated with doxycycline to silence ATF4 and then exposed to severe hypoxia. ATF4 was depleted at least $80 \%$ by this treatment, which caused a $50 \%$ reduction in ULK1 protein (Supplementary Figure S6C at http://www.biochemj.org/bj/449/bj4490389add. htm) and total immunoprecipitated ULK1 activity $(P<0.05)$ (Figure $3 \mathrm{E}$ ) after $36 \mathrm{~h}$ in severe hypoxia, despite no change in the specific activity of ULK1 (Supplementary Figure S6D).

\section{ULK1 is required for hypoxia-induced autophagy and mitophagy}

To investigate the requirement for ULK1 in hypoxia-induced autophagy, we depleted ULK1 using RNAi by $90 \%$ (Supplementary Figure S7A at http://www.biochemj.org/bj/449/ bj4490389add.htm), and exposed A431 and MCF7 cells to severe hypoxia. Indeed, knockdown of ULK1 severely reduced the accumulation of LC3-II protein (the lipidated form of LC3, a marker for autophagy) in A431 cells (Figure 4A) and to a lesser extent in MCF7 cells (Figure 4B) in severe hypoxia. 
Likewise, ULK1 knockdown resulted in the accumulation of p62 protein after $24 \mathrm{~h}$ in severe hypoxia, indicating that ablation of ULK1 resulted in reduced autophagic flux (Figures 4A and 4B). Knockdown of ATF4, was less consistent, and even resulted in increased levels of autophagy in some cases.

Staining revealed that ULK1 knockdown abrogated the formation of LC3-positive autophagosomes in both cell lines (Figures 4C and 4D). Likewise, cells depleted of ULK1 failed to accrue autophagosomes in the presence of chloroquine (an inhibitor of autophagosome fusion with lysosmes) (Supplementary Figure S7B), again indicating that ULK1 is required for the initiation of autophagy in severe hypoxia.

Furthermore, through the use of Imagestream ${ }^{\circledR}$ analysis of autophagic flux [31] (co-localization of endogenous LC3 protein and lysosomal stain), we found that ablation of ULK1 reduced flux in MCF7 cells by $90 \%$ in severe hypoxia $(P<0.001)$ and, intriguingly, by more than $80 \%$ in normoxia $(P<0.001)$ (Figures 4E and 4F). Thus ULK1 is involved in both basal autophagy and hypoxia-induced autophagy.

ULK1 has been shown previously to play an important role in the regulation of mitophagy in starvation and red blood cell maturation $[33,43]$. When mitochondrial mass was assessed by flow cytometry, it was found that depletion of ULK1 or ATF4 increased mean mitochondrial fluorescence by $40 \%$ and $20 \%$ respectively in both normoxia and hypoxia $(P<0.001)$ (Figure 4G), suggesting that ULK1 and ATF4 are required for both constitutive and hypoxia-induced mitophagy.

\section{ULK1 and ATF4 are required for growth and survival in normoxia and severe hypoxia}

Having established that ULK1 is required for autophagy and mitophagy in both normoxia and severe hypoxia, we next sought to assess the effects of their loss on cell death. Depletion of ULK1 by RNAi markedly increased AV staining in A431 and MCF7 cells in both normoxia and severe hypoxia $(P<0.001)$ (Figures $5 \mathrm{~A}$ and 5B). Depletion of ULK1 and ATF4 reduced clonogenicity by $65 \%$ and $80 \%$ respectively in both normoxia and severe hypoxia $(P<0.001)$ (Figure 5C). Likewise, knockdown of ULK1 or ATF4 in severe hypoxia reduced cellular ATP levels by more than $75 \%$ (Figure 5D).

To determine whether the effector caspases were activated by ablation of ULK1 or ATF4, caspase 3/7 activity was measured. Loss of ATF4 in A431 cells nearly doubled the observed caspase $3 / 7$ activity, indicating that apoptosis was being activated $(P<0.001)$ (Figure 5E). On the other hand, ULK1 knockdown actually reduced caspase $3 / 7$ activity in both normoxia and severe hypoxia $(P<0.01$ and $P<0.001)$ (Figure 5E), suggesting that cell death in this case is due to a caspase-3/7-independent mechanism, such a necrosis.

One common marker for necrosis is HMGB1, an architectural chromatin-binding factor [44-46]. Indeed, knockdown of ULK1 or ATF4 in A431 cells exposed to normoxia or severe hypoxia resulted in an increase in HMGB1 in the medium (Figure 5F) and decreased intracellular HMGB1 (Figure 5G). This result implied that knockdown of either ULK1 or ATF4 results in caspase-3/7independent cell death, although it is not possible to identify the mechanism as necrosis without further study.

Further evidence for non-apoptotic cell death was found when cell morphology was examined in spheroids. Spheroids derived from ATF4-knockdown cells showed a high degree of nuclear fragmentation and more caspase 3 cleavage than the scrambled control, whereas spheroids formed from the ULK1-knockdown cells showed almost no nuclear fragmentation and less caspase 3 cleavage than the scrambled control (Supplementary Figure S8A at http://www.biochemj.org/bj/449/bj4490389add.htm).

\section{Depletion of ULK1 or ATF4 reduces spheroid growth}

We next assessed the effect of ULK1 or ATF4 knockdown on growth in a three-dimensional spheroid model [34]. Spheroids derived from A431 cells depleted of ULK1 or ATF4 showed significantly retarded growth $(P<0.01)$ (Figures 6A and 6B). ULK1- and ATF4-depeleted spheroids harvested on day 3 contained 84 and $90 \%$ AV-positive cells respectively, whereas control spheroids contained $26 \%$ AV-positive cells $(P<0.01)$ (Figure 6C). Staining for pimonidazole, CAIX and CHOP indicated that the ULK1- and ATF4-knockdown spheroids were much less hypoxic than the scrambled controls (Figure 6D). Staining for Ki67, a marker of proliferation, showed no difference between groups (Figure 6D).

To determine whether a similar effect could be observed by pharmacological inhibition of autophagy, A431 spheroids were prepared and treated with a single dose of chloroquine or bafilomycin A1. Spheroids treated in this way showed a similar, although less complete, growth delay (Supplementary Figure S8B).

\section{High levels of ULK1 correlate with a poor prognosis in breast cancer}

To examine the potential importance of ULK1 in cancer prognosis and treatment, the expression of ULK1 mRNA was examined in a cohort of 152 breast cancer patients with long-term follow up. Univariate analysis revealed that patients with the highest level of $U L K 1 \mathrm{mRNA}$ had significantly lower relapse-free survival (log-rank test, $P=0.021$ ) (Figure 7). This association remained significant when Cox multivariate analysis including oestrogenreceptor status, patient age, nodal status, tumour size and tumour grade was performed $(P<0.034)$.

\section{DISCUSSION}

Hypoxia is a common feature of solid tumours, and is an essential determinant of prognosis and response to therapy. The activation of the ISR and autophagy represents a mechanism of cancer cell resistance to hypoxia. In the present study we demonstrated that the essential autophagy gene $U L K 1$ is a link between these two mechanisms. We showed that $U L K 1$ is transcriptionally upregulated in severe hypoxia and in response to ER stress by the direct activity of ATF4 at the $U L K 1$ promoter. Previous work has shown that ATF4 and CHOP can support high levels of autophagic flux in severe hypoxia through the transcriptional up-regulation of autophagic machinery proteins $M A P 1 L C 3 B$ and $A T G 5$, but does not support de novo autophagy itself $[19,21]$. Thus the results of the present study represents the first example in which ATF4 and the ISR are shown to regulate the expression of an autophagyinitiating factor and thus to potentially play an important role in the initiation of de novo autophagy.

It is well known that ULK1 kinase activity is subject to substantial post-translation regulation [47]. Indeed, to date most work has focused on the phosphorylation events that regulate changes in the specific activity of existing ULK1 protein. In the present study, we found that the total activity of ULK1 is also subject to substantial transcriptional regulation by ATF4. When we exposed A431 cells to severe hypoxia the increase in total ULK1 kinase activity was dependent on the transcriptional up-regulation of ULK1 by ATF4, although the specific activity of ULK1 remained unchanged, the massive accumulation of 
A

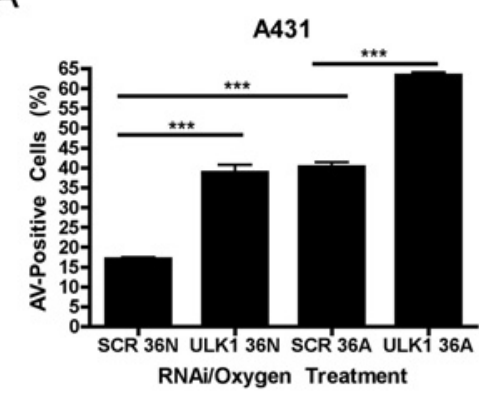

C A431

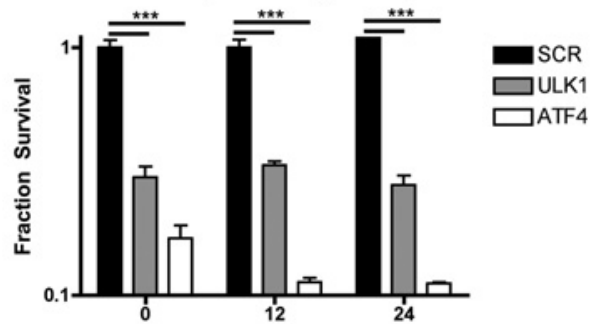

Time in Severe Hypoxia (HR)

E A431

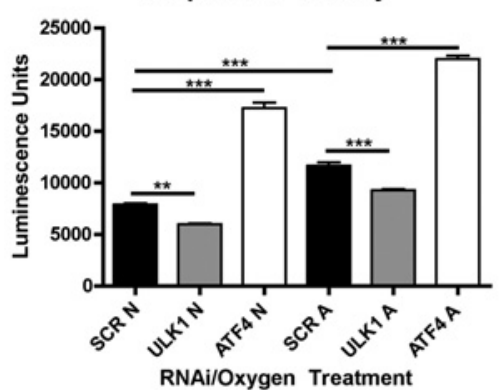

F A431

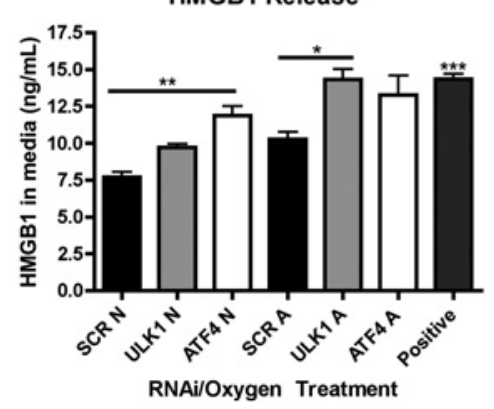

B

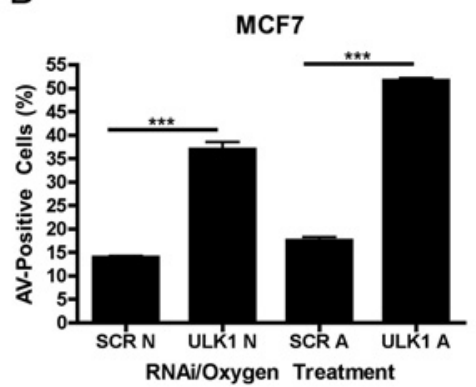

D A431

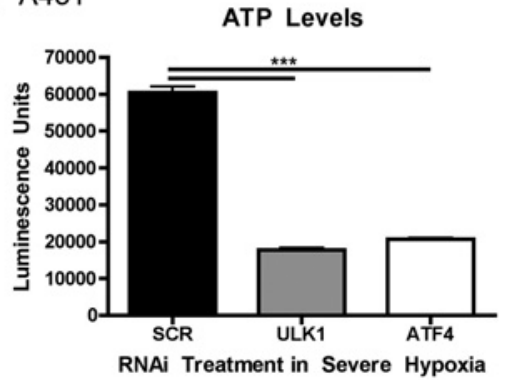

G

A431

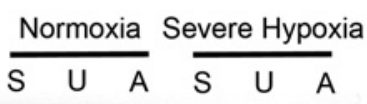

HMGB1

Actin

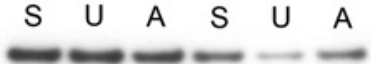

-

Figure 5 ULK1 and ATF4 are required for cancer cell survival in both normoxia and severe hypoxia

A431 (A and D-F) or MCF7 (B) cells were transfected with siRNA against ULK1, ATF4 or a scrambled control sequence (SCR) overnight and exposed to severe hypoxia (A) or normoxia (N) the next day. After $36 \mathrm{~h}, \mathrm{~A} 431(\mathbf{A})$ or MCF7 (B) cells were stained with Alexa Fluor ${ }^{\circledR}$-conjugated anti-AV antibody and analysed by flow cytometry. (C) Clonogenicity was assessed by seeding serial dilutions of A431 cells into $60 \mathrm{~mm}$ dishes and exposing them to severe hypoxia for 12 or $24 \mathrm{~h}$. (D) Changes in ATP in severe hypoxia were assessed after exposure to severe hypoxia for $36 \mathrm{~h}$. (E) Caspase $3 / 7$ activity was determined after $24 \mathrm{~h}$ in severe hypoxia. (F) HMGB1 protein in the medium was quantified by ELISA after $36 \mathrm{~h}$ in severe hypoxia. (G) Intracellular HMGB1 was quantified by Western blotting after $36 \mathrm{~h}$ in severe hypoxia. ${ }^{\star} P<0.05,{ }^{\star \star} P<0.01$ and ${ }^{\star \star \star} P<0.001$ respectively.

ULK1 protein resulted in greatly increased total kinase activity. In contrast, in MCF7 cells the specific kinase activity of ULK1 markedly increased without protein induction, suggesting that activity is regulated by post-translational mechanisms. In this context, ATF4 appeared to play a role in the maintenance of ULK1 protein level rather than hypoxic up-regulation. Thus, depending on cell type, either transcriptional up-regulation or post-translational phosphorylation may be the primary mode of regulating ULK1 kinase activity. In support of this notion, recent work has demonstrated that ULK1 expression and autophagy induction in response to DNA damage were increased through transactivation of ULK1 by p53 [48]. 


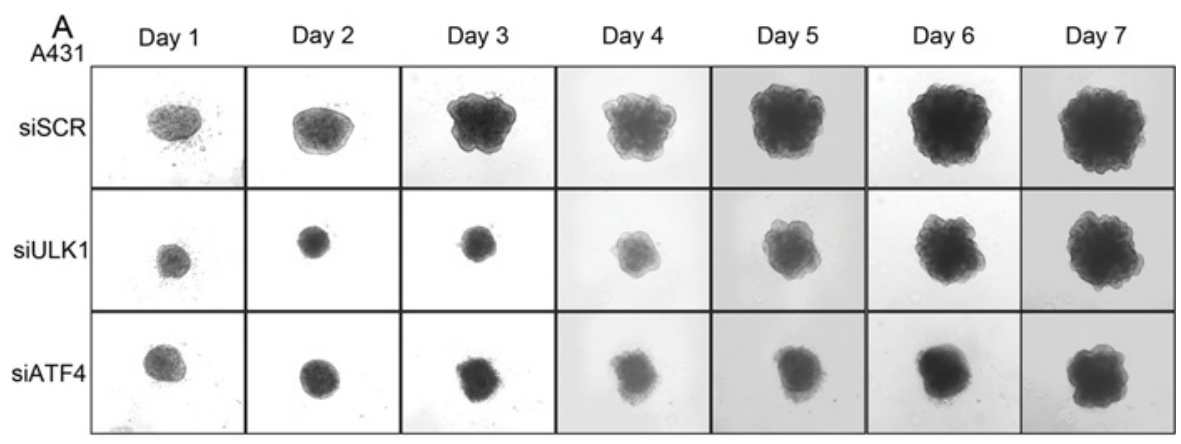

B

C
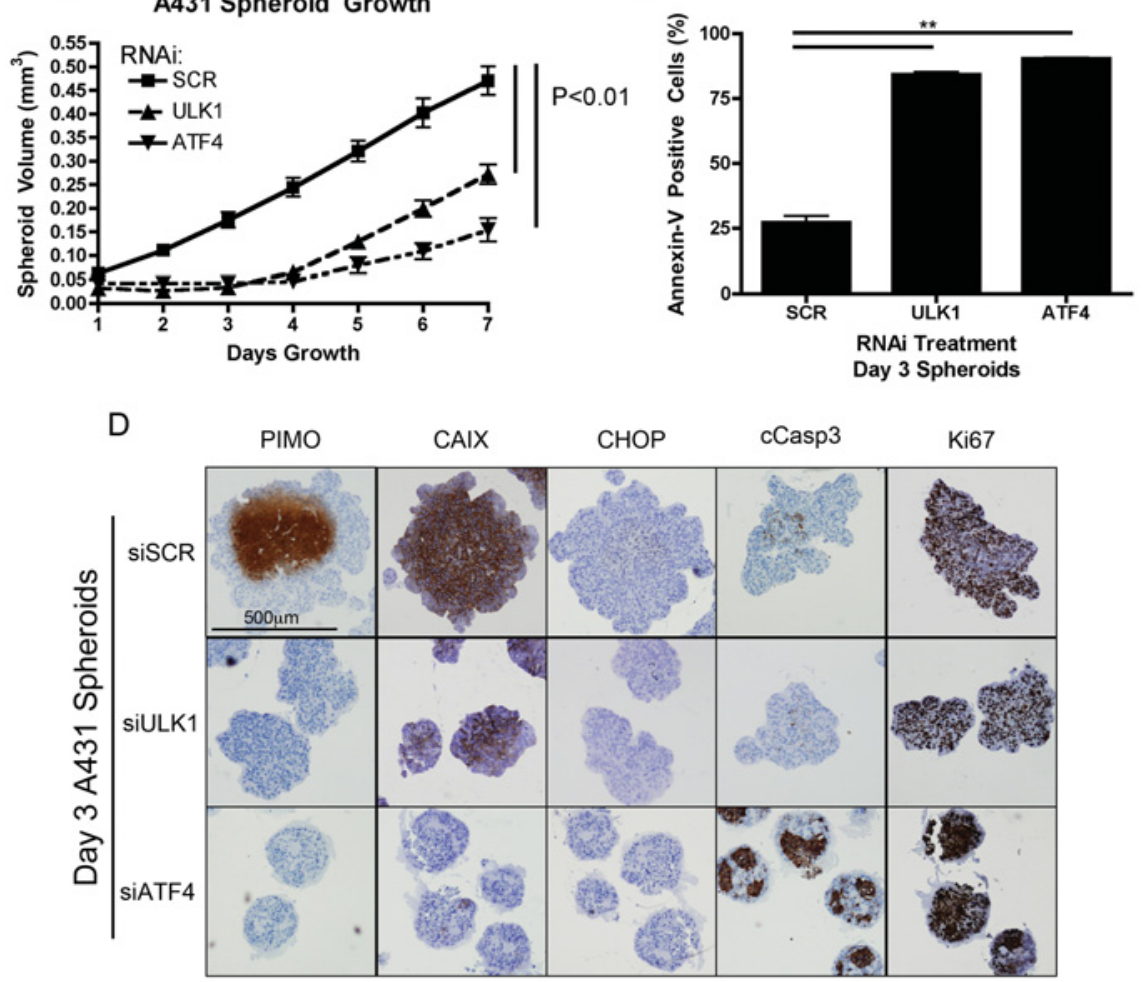

Figure 6 Knockdown of ULK1 or ATF4 reduces spheroid growth

A431 cells were transfected with siRNA sequences specific for ULK1, ATF4 or a scrambled control (SCR). The next day, cells were reseeded into round-bottomed 96 -well plates at 5000 cells/well. After $24 \mathrm{~h}$ spheroid-like structures formed and began to grow (Day 1). (A) Images were taken daily and volume measurements were calculated with ImageJ (NIH) $(\mathbf{B})$. Representative images from $n=4$ independent experiments are shown, with $n=10$ spheroids in each group for each experiment. Regression analysis was applied to calculate the slope of each curve and $P$ values were calculated by one-way ANOVA. (C) Spheroids were harvested on day 3 and dissociated using Accutase ${ }^{\circledR}$. Following staining with Alexa Fluor ${ }^{\circledR}$-conjugated anti-AV antibody, cells were analysed by flow cytometry. (D) Alternatively, day 3 spheroids were treated with pimonidazole (PIMO) for $3 \mathrm{~h}$ and then fixed and stained by immunohistochemistry using the antibodies indicated. cCasp3, cleaved caspase 3 .

In the present study, we demonstrated that ULK1 is required for autophagy in severe hypoxia, and ATF4 is responsible for increasing or maintaining ULK1 kinase activity in a celldependent context. We found that knockdown of ULK1 reduced autophagy induction in severe hypoxia when measured by Western blotting and immunofluorescence. To our surprise, when we examined autophagic flux by ImageStream ${ }^{\circledR}$ analysis, we found that this dependence also occurred in normoxic conditions, and that ablation of ULK1 or ATF4 in normoxia, severe hypoxia or three-dimensional cell culture (spheroids) resulted in a similar reduction in autophagic flux, and a similar enhancement of cell death. Furthermore, RNAi against ATF4 reduced ULK1 levels in normoxia as well as hypoxia, suggesting that both ATF4 and ULK1 are active in the 'unperturbed' normoxic condition. In this vein, it has become apparent that autophagy is crucial for cancer cell growth and the survival of the oncogenic changes that lead to cancer development-persistent autophagy is necessary to maintain a viable pool of mitochondria in H-Ras-expressing cancer cells and pancreatic cancer cells under otherwise unstressed conditions, a phenomenon that has been termed 'autophagy addiction' $[26,27,49,50]$. Importantly, it appears that ULK1 is dispensable in untransformed cells under normal growth conditions and is necessary for survival only in starvation conditions. ULK1knockout mice are viable, showing only mild defects in haemopoesis [33]. Furthermore, ULK1 ${ }^{-1-}$ MEFs (mouse embryonic fibroblasts) expressing a stable shRNA construct against ULK2 are viable under normal growth conditions. It is only when these cells are starved of amino acids and serum that the ULK1/ULK2 deficiency results in cell death [43].

Previous work has shown that autophagy inhibition can cause necrosis in apoptosis-deficient cells [25]. Alternatively, there are a number of systems in which inhibition of autophagy can lead to 


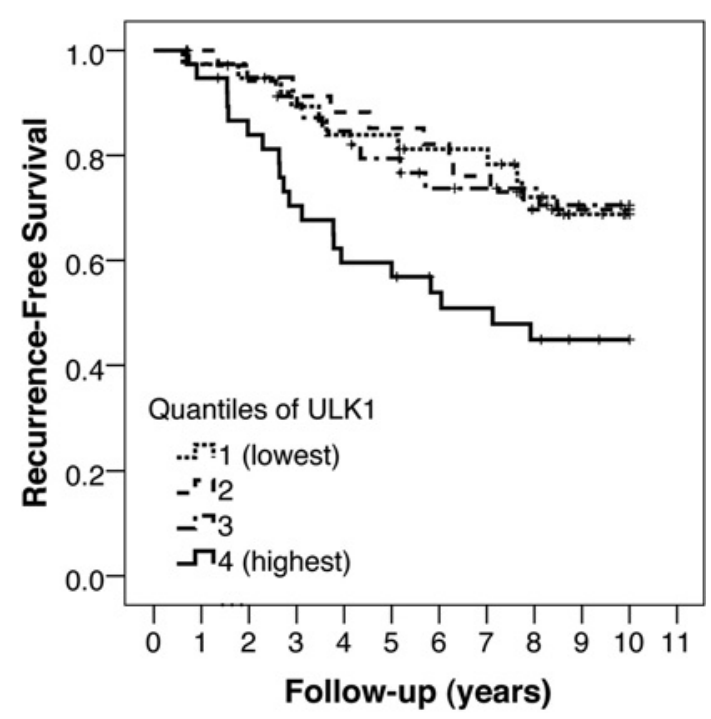

Figure 7 High levels of ULK1 correlate with a poor prognosis in breast cancer

ULK1 gene expression was considered by univariate analysis and patient samples were ranked from low to high expression, normalized from 0 to 1 , and divided into quartiles. High ULK1 expression correlated with decreased recurrence-free survival. Analyses were performed using the Spearman's Rank correlation test and ANOVA. $n=152$.

a reduction in apoptosis or cell death [28,48,51-55]. Moreover, recent work has implicated ULK1/ATG13-mediated autophagy in promoting DNA-damage-induced cell death, although the authors failed to show a direct role for ULK1 in this process [48]. In the present study, we found that ablation of endogenous ULK1 predisposed cells to die by an unidentified caspase3/7-independent mechanism, whereas loss of ATF4 resulted in apoptotic cell death. One explanation for this result could be that autophagy is required for the maintenance of a low level of ATP to initiate caspase activation. Previous work has indicated that mitophagy is required for apoptosis induction in certain contexts [52]. Alternatively, it may also be that loss of ULK1 results in elevated ROS (reactive oxygen species) and lysosomal dysfunction, leading to necroptosis [56-58]. In either scenario, the residual pool of ULK1 remaining following ATF4 knockdown might be sufficient to drive caspase-dependent death. In any case, further study is necessary to identify the mechanism of this caspase-3/7-independent cell death.

We found a positive correlation between $U L K 1 \mathrm{mRNA}$ levels and reduced relapse-free survival in a cohort of breast cancer patients, implying that ULK1 up-regulation may be a mode of breast cancer cell survival and tumour progression. Likewise, the ISR is important in cancer cell survival and tumour maintenance. Tumour xenografts deficient in ATF4 or expressing a dominantnegative construct of eIF $2 \alpha$ show greatly retarded growth and enhanced levels of cell death [19,23,24]. Data indicate that ATF4 and the ISR promote cancer cell survival of hypoxia, nutrient deprivation and oxidative stress in vitro [9,19-22], in part through the maintenance of high levels of autophagic flux through the upregulation of $M A P 1 L C 3 B$ and $A T G 5$ [19,21]. The present study demonstrates for the first time that ATF4 can transcriptionally up-regulate the autophagy-initiating kinase ULK1 and that ULK1 is crucial for autophagy, mitophagy and cancer cell survival in normoxia, severe hypoxia and spheroids. Thus, on the basis of the results of the present study and those of others [26,27], we speculate that a specific ULK1 inhibitor may be an effective killing agent against a subset of cancers, with limited toxicity towards normal cells.

\section{AUTHOR CONTRIBUTION}

Luke Pike conceived and conducted all experiments unless otherwise specified. Adrian Harris supervised the project and research group. Dean Singleton assisted with Western blots for LC3 in Figures 1 and 4, as well as experiments pertaining to viral shRNA knockdown of ULK1. Francesca Buffa prepared Figure 7 in its entirety. Olga Abramczyk and James Murray conducted ULK1 kinase activity experiments. Kanchan Phadwal and Anna Katharina Simon conducted Imagestream analysis of autophagy. Ji-Liang Li conducted tumour xenograft studies with bevacizumab treatment, from which RNA was extracted and analysed.

\section{ACKNOWLEDGEMENTS}

We thank Dr Karim Bensaad and Dr Alan MacIntyre for their critical reading of this work before submission.

\section{FUNDING}

This work was supported by Cancer Research UK, the Rhodes Trust, the NIHR (National Institute for Health Research) Oxford Biomedical Research Centre, and the Natural Sciences and Engineering Research Council of Canada.

\section{REFERENCES}

1 Thomlinson, R. H. and Gray, L. H. (1955) The histological structure of some human lung cancers and the possible implications for radiotherapy. Br. J. Cancer $\mathbf{9}, 539-549$

2 Bertout, J. A., Patel, S. A. and Simon, M. C. (2008) The impact of $\mathrm{O}_{2}$ availability on human cancer. Nat. Rev. Cancer 8, 967-975

3 Olive, P. L., Banath, J. P. and Durand, R. E. (2002) The range of oxygenation in SiHa tumor xenografts. Radiat. Res. 158, 159-166

4 Hockel, M., Schlenger, K., Aral, B., Mitze, M., Schaffer, U. and Vaupel, P. (1996) Association between tumor hypoxia and malignant progression in advanced cancer of the uterine cervix. Cancer Res. 56, 4509-4515

5 Hockel, M., Vorndran, B., Schlenger, K., Baussmann, E. and Knapstein, P. G. (1993) Tumor oxygenation: a new predictive parameter in locally advanced cancer of the uterine cervix. Gynecol. Oncol. 51, 141-149

6 Milani, M. and Harris, A. L. (2008) Targeting tumour hypoxia in breast cancer. Eur. J. Cancer 44, 2766-2773

7 Harris, A. L. (2002) Hypoxia: a key regulatory factor in tumour growth. Nat. Rev. Cancer 2 $38-47$

8 Tu, B. P. and Weissman, J. S. (2002) The FAD- and $\mathrm{O}_{2}$-dependent reaction cycle of Ero1-mediated oxidative protein folding in the endoplasmic reticulum. Mol. Cell 10 983-994

9 Harding, H. P., Zhang, Y., Zeng, H., Novoa, I., Lu, P. D., Calfon, M., Sadri, N., Yun, C., Popko, B., Paules, R. et al. (2003) An integrated stress response regulates amino acid metabolism and resistance to oxidative stress. Mol. Cell 11, 619-633

10 Bernales, S., Papa, F. R. and Walter, P. (2006) Intracellular signaling by the unfolded protein response. Annu. Rev. Cell Dev. Biol. 22, 487-508

11 Harding, H. P., Novoa, I., Zhang, Y., Zeng, H., Wek, R., Schapira, M. and Ron, D. (2000) Regulated translation initiation controls stress-induced gene expression in mammalian cells. Mol. Cell 6, 1099-1108

12 Harding, H. P., Zhang, Y. and Ron, D. (1999) Protein translation and folding are coupled by an endoplasmic-reticulum-resident kinase. Nature $\mathbf{3 9 7}, 271-274$

13 Liang, G. and Hai, T. (1997) Characterization of human activating transcription factor 4, a transcriptional activator that interacts with multiple domains of CAMP-responsive element-binding protein (CREB)-binding protein. J. Biol. Chem. 272, 24088-24095

14 Blais, J. D., Filipenko, V., Bi, M., Harding, H. P., Ron, D., Koumenis, C., Wouters, B. G. and Bell, J. C. (2004) Activating transcription factor 4 is translationally regulated by hypoxic stress. Mol. Cell. Biol. 24, 7469-7482

15 Bobrovnikova-Marjon, E., Grigoriadou, C., Pytel, D., Zhang, F., Ye, J., Koumenis, C., Cavener, D. and Diehl, J. A. (2010) PERK promotes cancer cell proliferation and tumor growth by limiting oxidative DNA damage. Oncogene 29, 3881-3895

16 Bobrovnikova-Marjon, E., Hatzivassiliou, G., Grigoriadou, C., Romero, M., Cavener, D. R., Thompson, C. B. and Diehl, J. A. (2008) PERK-dependent regulation of lipogenesis during mouse mammary gland development and adipocyte differentiation. Proc. Natl. Acad. Sci. U.S.A. 105, 16314-16319 
17 Yoshizawa, T., Hinoi, E., Jung, D. Y., Kajimura, D., Ferron, M., Seo, J., Graff, J. M., Kim, J. K. and Karsenty, G. (2009) The transcription factor ATF4 regulates glucose metabolism in mice through its expression in osteoblasts. J. Clin. Invest. 119, 2807-2817

18 Ghosh, R., Lipson, K. L., Sargent, K. E., Mercurio, A. M., Hunt, J. S., Ron, D. and Urano, F. (2010) Transcriptional regulation of VEGF-A by the unfolded protein response pathway. PLOS ONE 5, e9575

19 Rouschop, K. M., van den Beucken, T., Dubois, L., Niessen, H., Bussink, J., Savelkouls, K., Keulers, T., Mujcic, H., Landuyt, W., Voncken, J. W. et al. (2009) The unfolded protein response protects human tumor cells during hypoxia through regulation of the autophagy genes MAP1LC3B and ATG5. J. Clin. Invest. 120, 127-141

20 Rzymski, T. and Harris, A. L. (2007) The unfolded protein response and integrated stress response to anoxia. Clin. Cancer Res. 13, 2537-2540

21 Rzymski, T., Milani, M., Pike, L., Buffa, F., Mellor, H. R., Winchester, L., Pires, I., Hammond, E., Ragoussis, I. and Harris, A. L. (2010) Regulation of autophagy by ATF4 in response to severe hypoxia. Oncogene $\mathbf{2 9}, 4424-4435$

22 Rouschop, K. M., Ramaekers, C. H., Schaaf, M. B., Keulers, T. G., Savelkouls, K. G., Lambin, P., Koritzinsky, M. and Wouters, B. G. (2009) Autophagy is required during cycling hypoxia to lower production of reactive oxygen species. Radiother. Oncol. 92 $411-416$

23 Bi, M., Naczki, C., Koritzinsky, M., Fels, D., Blais, J., Hu, N., Harding, H., Novoa, I., Varia, M., Raleigh, J. et al. (2005) ER stress-regulated translation increases tolerance to extreme hypoxia and promotes tumor growth. EMBO J. 24, 3470-3481

24 Ye, J., Kumanova, M., Hart, L. S., Sloane, K., Zhang, H., De Panis, D. N., Bobrovnikova-Marjon, E., Diehl, J. A., Ron, D. and Koumenis, C. (2010) The GCN2-ATF4 pathway is critical for tumour cell survival and proliferation in response to nutrient deprivation. EMBO J. 29, 2082-2096

25 Degenhardt, K., Mathew, R., Beaudoin, B., Bray, K., Anderson, D., Chen, G. Mukherjee, C., Shi, Y., Gelinas, C., Fan, Y. et al. (2006) Autophagy promotes tumor cell survival and restricts necrosis, inflammation, and tumorigenesis. Cancer Cell 10, 51-64

26 Yang, S., Wang, X., Contino, G., Liesa, M., Sahin, E., Ying, H., Bause, A., Li, Y., Stommel, J. M., Dell'antonio, G. et al. (2011) Pancreatic cancers require autophagy for tumor growth. Genes Dev. 25, 717-729

27 Guo, J. Y., Chen, H. Y., Mathew, R., Fan, J., Strohecker, A. M., Karsli-Uzunbas, G., Kamphorst, J. J., Chen, G., Lemons, J. M., Karantza, V. et al. (2011) Activated Ras requires autophagy to maintain oxidative metabolism and tumorigenesis. Genes Dev. 25, 460-470

28 Elgendy, M., Sheridan, C., Brumatti, G. and Martin, S. J. (2011) Oncogenic Ras-induced expression of Noxa and Beclin-1 promotes autophagic cell death and limits clonogenic survival. Mol. Cell 42, 23-35

29 Milani, M., Rzymski, T., Mellor, H. R., Pike, L., Bottini, A., Generali, D. and Harris, A. L. (2009) The role of ATF4 stabilization and autophagy in resistance of breast cancer cells treated with Bortezomib. Cancer Res. 69, 4415-4423

30 Rzymski, T., Paantjens, A., Bod, J. and Harris, A. L. (2008) Multiple pathways are involved in the anoxia response of SKIP3 including HuR-regulated RNA stability, NF- $\kappa$ B and ATF4. Oncogene 27, 4532-4543

31 Phadwal, K., Alegre-Abarrategui, J., Watson, A. S., Pike, L., Anbalagan, S., Hammond, E. M., Wade-Martins, R., McMichael, A., Klenerman, P. and Simon, A. K. (2012) A novel method for autophagy detection in primary cells: impaired levels of macroautophagy in immunosenescent T cells. Autophagy 8, 677-689

32 Otsuki, Y., Li, Z. and Shibata, M. A. (2003) Apoptotic detection methods: from morphology to gene. Prog. Histochem. Cytochem. 38, 275-339

33 Kundu, M., Lindsten, T., Yang, C. Y., Wu, J., Zhao, F., Zhang, J., Selak, M. A., Ney, P. A. and Thompson, C. B. (2008) Ulk1 plays a critical role in the autophagic clearance of mitochondria and ribosomes during reticulocyte maturation. Blood 112, 1493-1502

34 Ivascu, A. and Kubbies, M. (2006) Rapid generation of single-tumor spheroids for high-throughput cell function and toxicity analysis. J. Biomol. Screening 11, 922-932

35 Higgins, G. S., Harris, A. L., Prevo, R., Helleday, T., McKenna, W. G. and Buffa, F. M. (2010) Overexpression of POLQ confers a poor prognosis in early breast cancer patients. Oncotarget 1, 175-184

36 Koumenis, C., Naczki, C., Koritzinsky, M., Rastani, S., Diehl, A., Sonenberg, N., Koromilas, A. and Wouters, B. G. (2002) Regulation of protein synthesis by hypoxia via activation of the endoplasmic reticulum kinase PERK and phosphorylation of the translation initiation factor elF2 $\alpha$. Mol. Cell. Biol. 22, 7405-7416
37 Paez-Ribes, M., Allen, E., Hudock, J., Takeda, T., Okuyama, H., Vinals, F., Inoue, M., Bergers, G., Hanahan, D. and Casanovas, 0. (2009) Antiangiogenic therapy elicits malignant progression of tumors to increased local invasion and distant metastasis. Cancer Cell 15, 220-231

38 Casanovas, 0., Hicklin, D. J., Bergers, G. and Hanahan, D. (2005) Drug resistance by evasion of antiangiogenic targeting of VEGF signaling in late-stage pancreatic islet tumors. Cancer Cell 8, 299-309

39 Ganley, I. G., Lam du, H., Wang, J., Ding, X., Chen, S. and Jiang, X. (2009) ULK1.ATG13.FIP200 complex mediates mTOR signaling and is essential for autophagy. J. Biol. Chem. 284, 12297-12305

40 Shang, L., Chen, S., Du, F., Li, S., Zhao, L. and Wang, X. (2011) Nutrient starvation elicits an acute autophagic response mediated by Ulk1 dephosphorylation and its subsequent dissociation from AMPK. Proc. Natl. Acad. Sci. U.S.A. 108, 4788-4793

41 Lee, J. W., Park, S., Takahashi, Y. and Wang, H. G. (2010) The association of AMPK with ULK1 regulates autophagy. PLOS ONE 5, e15394

42 Kim, J., Kundu, M., Viollet, B. and Guan, K. L. (2011) AMPK and mTOR regulate autophagy through direct phosphorylation of Ulk1. Nat. Cell Biol. 13, 132-141

43 Egan, D. F., Shackelford, D. B., Mihaylova, M. M., Gelino, S., Kohnz, R. A., Mair, W. Vasquez, D. S., Joshi, A., Gwinn, D. M., Taylor, R. et al. (2011) Phosphorylation of ULK1 (hATG1) by AMP-activated protein kinase connects energy sensing to mitophagy. Science 331, 456-461

44 Scaffidi, P., Misteli, T. and Bianchi, M. E. (2002) Release of chromatin protein HMGB1 by necrotic cells triggers inflammation. Nature 418, 191-195

45 Muller, S., Scaffidi, P., Degryse, B., Bonaldi, T., Ronfani, L., Agresti, A., Beltrame, M. and Bianchi, M. E. (2001) The double life of HMGB1 chromatin protein: architectural factor and extracellular signal. EMBO J. 20, 4337-4340

46 Bustin, M. (1999) Regulation of DNA-dependent activities by the functional motifs of the high-mobility-group chromosomal proteins. Mol. Cell. Biol. 19, 5237-5246

47 Mizushima, N. (2010) The role of the Atg1/ULK1 complex in autophagy regulation. Curr. Opin. Cell Biol. 22, 132-139

48 Gao, W., Shen, Z., Shang, L. and Wang, X. (2011) Upregulation of human autophagy-initiation kinase ULK1 by tumor suppressor p53 contributes to DNA-damage-induced cell death. Cell Death Differ. 18, 1598-1607

49 Mancias, J. D. and Kimmelman, A. C. (2011) Targeting autophagy addiction in cancer. Oncotarget 2, 1302-1306

50 Rabinowitz, J. D. and White, E. (2011) Autophagy and metabolism. Science 330 1344-1348

51 Zhu, J. H., Horbinski, C., Guo, F., Watkins, S., Uchiyama, Y. and Chu, C. T. (2007) Regulation of autophagy by extracellular signal-regulated protein kinases during 1-methyl-4-phenylpyridinium-induced cell death. Am. J. Pathol. 170, 75-86

52 Yee, K. S., Wilkinson, S., James, J., Ryan, K. M. and Vousden, K. H. (2009) PUMAand Bax-induced autophagy contributes to apoptosis. Cell Death Differ. 16, $1135-1145$

53 Xue, L., Fletcher, G. C. and Tolkovsky, A. M. (1999) Autophagy is activated by apoptotic signalling in sympathetic neurons: an alternative mechanism of death execution. Mol. Cell. Neurosci. 14, 180-198

54 Crighton, D., Wilkinson, S., O'Prey, J., Syed, N., Smith, P., Harrison, P. R., Gasco, M., Garrone, 0., Crook, T. and Ryan, K. M. (2006) DRAM, a p53-induced modulator of autophagy, is critical for apoptosis. Cell 126, 121-134

55 Berry, D. L. and Baehrecke, E. H. (2007) Growth arrest and autophagy are required for salivary gland cell degradation in Drosophila. Cell 131, 1137-1148

56 Vandenabeele, P., Galluzzi, L., Vanden Berghe, T. and Kroemer, G. (2010) Molecular mechanisms of necroptosis: an ordered cellular explosion. Nat. Rev. Mol. Cell Biol. 11, 700-714

57 Zhang, N., Chen, Y., Jiang, R., Li, E., Chen, X., Xi, Z., Guo, Y., Liu, X., Zhou, Y., Che, Y. and Yue, $X$. (2011) PARP and RIP 1 are required for autophagy induced by $11^{\prime}$-deoxyverticillin A, which precedes caspase-dependent apoptosis. Autophagy 7, 598-612

58 Bonapace, L., Bornhauser, B. C., Schmitz, M., Cario, G., Ziegler, U., Niggli, F. K., Schafer, B. W., Schrappe, M., Stanulla, M. and Bourquin, J. P. (2010) Induction of autophagy-dependent necroptosis is required for childhood acute lymphoblastic leukemia cells to overcome glucocorticoid resistance. J. Clin. Invest. 120 $1310-1323$ 


\title{
SUPPLEMENTARY ONLINE DATA
}

\section{Transcriptional up-regulation of ULK1 by ATF4 contributes to cancer cell survival}

\author{
Luke R. G. PIKE*, Dean C. SINGLETON, Francesca BUFFA*, Olga ABRAMCZYK†, Kanchan PHADWAL + , Ji-Liang LI*, \\ Anna Katharina SIMON $\neq$, James T. MURRAY $\S$ and Adrian L. HARRIS*1 \\ *Growth Factor Group, Cancer Research UK, Molecular Oncology Laboratories, Weatherall Institute of Molecular Medicine, University of Oxford, John Radcliffe Hospital, Headington, \\ Oxford, U.K., † †entre for Cancer Research and Cell Biology, Queen's University Belfast, CCRCB Building, Belfast, U.K., ¥Nuffield Department of Medicine, NIHR, Biomedical Research \\ Centre, John Radcliffe Hospital, Headington, Oxford, U.K., and §School of Biochemistry and Immunology, Trinity Biomedical Sciences Institute, Room 5.50, 152-160 Pearse Street, \\ Trinity College Dublin, Dublin 2, Ireland
}

\section{MATERIALS AND METHODS}

\section{Antibodies}

Rabbit anti-ULK1 (R600), rabbit anti-ACC, rabbit anti-HMGB1, rabbit anti-p70S6K, rabbit anti-mTOR, rabbit anti-P-ACC $\left(\operatorname{Ser}^{79}\right)$, rabbit anti-P-AMPK $\alpha$ (Thr $\left.{ }^{172}\right)$, rabbit anti-phospho-p70S6K $\left(\mathrm{Ser}^{371}\right)$, rabbit anti-P-Raptor $\left(\mathrm{Ser}^{792}\right)$ and rabbit anti-Raptor were obtained from Cell Signaling Technology. A rabbit polyclonal antibody against ATF4 was obtained from Santa Cruz Biotechnology. The mouse monoclonal antibody against MAP1LC3 was purchased from NanoTools. Rabbit anti-HIF2 $\alpha$ antibody was obtained from AbD Serotec. Mouse anti- $\beta$-actin was obtained from Sigma-Aldrich. Mouse anti-CHOP was obtained from Santa Cruz Biotechnology. Mouse anti-CAIX was prepared in-house by Cancer Research UK. Rabbit anti-cleaved caspase 3 was obtained from R\&D Systems. Mouse anti-pimonidazole was obtained from Chemicon International.

\section{ULK1 activity assays}

\section{Anti-ULK1 antibody}

A specific antibody was raised, in sheep, against a peptide corresponding to a region between the $\mathrm{N}$-terminal kinase domain and C-terminal-binding domain coupled to KLH (keyhole-limpet haemocyanin). Antibodies were affinity-purified against peptide antigen before use.

\section{Immunoprecipitation of ULK1}

Extracts were prepared in lysis buffer $[50 \mathrm{mM}$ Tris/ $\mathrm{HCl}$ (pH 7.5), $1 \mathrm{mM}$ EGTA, $1 \mathrm{mM}$ EDTA, $0.3 \%$ CHAPS, $1 \mathrm{mM}$ sodium orthovanadate, $50 \mathrm{mM}$ sodium fluoride, $5 \mathrm{mM}$ sodium pyrophosphate, $0.27 \mathrm{M}$ sucrose and $0.1 \%$ 2-mercaptoethanol] and clarified by centrifugation at $16000 \mathrm{~g}$ for $20 \mathrm{~min}$ at $4{ }^{\circ} \mathrm{C}$. To precipitate ULK1, anti-ULK1 antibody was added to cell extracts and subjected to overnight incubation at $4{ }^{\circ} \mathrm{C}$ on a Vibrax shaker. Immunocomplexes were captured on Protein GSepharose (GE Lifesciences) and incubated for a further $1 \mathrm{~h}$ at $4{ }^{\circ} \mathrm{C}$ on a Vibrax shaker. Complexes were washed once in highsalt buffer (lysis buffer containing $500 \mathrm{mM} \mathrm{NaCl}$ ), twice in lysis buffer and twice in assay buffer [20 mM Hepes (pH 7.5), $150 \mathrm{mM}$ $\mathrm{NaCl}, 0.1 \%$ 2-mercaptoethanol, $25 \mathrm{mM}$ 2-glycerophosphate and $100 \mu \mathrm{M}$ sodium orthovanadate], before finally resuspending in a final volume of $24 \mu \mathrm{l}$ of assay buffer.

\section{ULK1 activity assay}

ULK1 activity was assayed at $30^{\circ} \mathrm{C}$ in a final reaction volume of $30 \mu \mathrm{l}$, containing $10 \mathrm{mM}$ magnesium acetate, $100 \mu \mathrm{M}[\gamma$ ${ }^{32}$ P]ATP (100-1000 c.p.m./pmol) against $3 \mu \mathrm{g}$ of MBP. Reactions were stopped after $30 \mathrm{~min}$ by addition of $10 \mu \mathrm{l}$ of $4 \times$ SDS sample buffer and samples were resolved by $8.0 \%$ Bis-Tris SDS/PAGE. The upper portion of each gel, containing immunoprecipitated ULK1, was immunoblotted on to Immobilon-P (Millipore) and probed for ULK1 precipitation. The lower portion of each gel containing PMBP was imaged by phosphoimaging using an FLA7000 PhosphoImager system (Fujifilm UK) and quantified using MultiGauge software.

\section{Imagestream ${ }^{\circledR}$ analysis of autophagy}

Imagestream ${ }^{\circledR}$ (IS100) is a multispectral flow cytometer combining standard microscopy with flow cytometry (Cronus Technology). It can acquire up to 100 cells/s, simultaneously acquiring six images of each cell, including brightfield, scatter and multiple fluorescent images. Imagestream ${ }^{\circledR}$ was used to analyse autophagic flux. In brief, MCF7 cells transfected with siRNA for ULK1 or scrambled sequence were exposed to severe hypoxia for $24 \mathrm{~h}$. At this point, cells were trypsinized and collected in $15 \mathrm{ml} \mathrm{Falcon}{ }^{\circledR}$ tubes. Following washes with assay buffer ( $1 \%$ FBS/PBS), cells were stained with a Violet live/dead marker (Invitrogen, L34955 Component A) and a red lysosome-specific dye (LysoID, Enzo Life Sciences). Cells were then fixed and permeabilized (eBioscience Fixation and Permeabilization kit) and stained with mouse anti-LC3 (Nanotools) and Alexa Fluor ${ }^{\circledR}$ 488-conjugated goat anti-mouse IgG (Invitrogen). Cells were imaged with the Imagestream ${ }^{\circledR}$ apparatus, and images were acquired for each respective channel. Single-stained controls were included for compensation. Following image acquisition, live cells were analysed for co-localization of LC3 and lysosomes using the software package IDEAS 4.0.735. Autophagy levels were calculated by measuring the percentage co-localization of LC3 and LysoID double-positive cells by plotting these LC3 + Lyso + (double-positives) for BDS (bright detailed similarity) between LC3 and lysosomal markers against normalized frequency of cells. This analysis therefore gave an estimation of the relative number of autophagolysosomes per cell, in a sample of more than 5000 cells, yielding a powerful assessment of autophagic flux.

\section{Patient details}

Tumour samples for 152 individuals were obtained from a retrospective series of patients with early primary breast

\footnotetext{
1 To whom correspondence should be addressed (email aharris.lab@imm.ox.ac.uk).
} 


\section{Table S1 siRNA sequences used for RNAi}

siRNA sequences were ordered from Eurogentec and diluted in RNAse-free water to prepare $100 \mu \mathrm{M}$ stocks. Working solutions were prepared to a final RNA concentration of $20 \mu \mathrm{M}$. These were diluted $1000 \times$ in the preparation of lipid constructs for a final concentration of $20 \mathrm{nM}$ RNA on cells. BNIP3, Bcl2/adenovirus E1B 19 kDa interacting protein 3.

\begin{tabular}{lll}
\hline Gene & Sense sequence $\left(5^{\prime} \rightarrow 3^{\prime}\right)$ & Working concentration \\
\hline ATF4 & CCACGUUGGAUGACACUUG & $20 \mathrm{nM}$ \\
Scrambled control (BNIP3 SCR) & ACGCGACACGCAGGUCGUCAU & $20 \mathrm{nM}$ \\
ULK1 & & \\
$\# 1$ & GCACAGAGACCGTGGGCAA & $5 \mathrm{nM}$ \\
$\# 2$ & CCACGCAGGTGCAGAACTA & $5 \mathrm{nM}$ \\
$\# 3$ & CGGAGAGCCTGCAGGAGA & $5 \mathrm{nM}$ \\
$\# 4$ & GAGCAAGAGCACACGGAGA & $5 \mathrm{nM}$ \\
& & \\
\hline
\end{tabular}

\section{Table S2 Primers for RT-qPCR}

Primers used for RT-qPCR designed using the Universal Probe Library primer design website (Roche).

\begin{tabular}{lll}
\hline Gene & Forward primer $\left(5^{\prime} \rightarrow 3^{\prime}\right)$ & Reverse primer $\left(5^{\prime} \rightarrow 3^{\prime}\right)$ \\
\hline CHOP & AAGGCACTGAGCGTATCATGT & TGAAGATACACTTCCTTCTTGAACA \\
Tubulin $\alpha 6$ (TUBA6) & CCCCTTCAGTTCTAGTCATGC & ATTGCCAATCTGGACACCA \\
ULK1 & TCATCTTCAGCCACGCTGT & CACGGTGCTGGAACATCTC \\
& & \\
\hline
\end{tabular}

cancer who were treated in Oxford, UK between 1990 and 1992. Patients received adjuvant chemotherapy and/or adjuvant hormone therapy, or no adjuvant treatment. This series is part of a published series, and full treatment details and demographics has been described previously [1].

\section{RNA extraction and gene expression profiling}

Total RNA was isolated using the TRIzol ${ }^{\circledR}$ method (Invitrogen) according to the manufacturer's instructions. mRNA expression was measured using Affimetrix U133 arrays. RNA was amplified using the Ambion Illumina Amplification kit. The full protocol
Table S3 Primers for ChIP

RT-qPCR primers designed against genomic regions of the ULK1 promoter, against an upstream region of chromosome 12 and for the promoter ATF4-target CHOP.

\begin{tabular}{lll}
\hline Primer set & Forward primer $\left(5^{\prime} \rightarrow 3^{\prime}\right)$ & Reverse primer $\left(5^{\prime} \rightarrow 3^{\prime}\right)$ \\
\hline CH0P & AGCCAAAATCAGAGCTGGAA & ACAAGTTGGCAAGCTGGTCT \\
CHR12 & GGGGCCATTTAAAGAGTAGTCGT & CCTTGTAAAACCATCAGTCGTCA \\
ULK1\#1 & GTGCGCTGCTTGGCCTAAGTGATG & ATCCGCTGGGGAGGAAGGTTAGC \\
ULK1 \#2 & CCAGCGGATTATGGGCTATGTCG & CCGCCCCCACTGCCTGTTCTCCTC \\
ULK1 \#3 & AAGGGGAAAGGAGGGAGGAGGA & GAGCCGGGCGTGACGACA \\
ULK1\#4 & CTGCGCGGGCGTCTCAG & GGCGAGGGCGCATCTCC \\
ULK1\#5 & GCGCCTCCGCCTGAGTCC & ATCTCGGGGCGGGGATGC \\
ULK1\#6 & CCAGCCCGACTTTCCTGTCCA & CTCCCCCAGACCCCAGTCCA \\
& & \\
\hline
\end{tabular}

has been described previously [1]. Data were pre-processed using gcrma, quantile normalized and logged (base 2) in Bioconductor (http://www.bioconductor.org). The target sequence of the probes that corresponded to ULK1 expression was retrieved using Affymetrix annotation and was 209333_at.

\section{Survival analysis}

The end point was relapse-free survival. Univariate and multivariate analysis was performed. Cox multivariate models were reduced using step-wise backward likelihood selection. In univariate analyses, expression of $U L K 1$ and other genes was considered either as divided in equal quartiles or as continuous variable ranked, and normalized between 0 and 1 . In multivariate analysis the latter was always considered.

\section{Animal experimentation}

All animal experiments were conducted according to Home Office regulations. When a tumour reached the maximum size $\left(1.44 \mathrm{~cm}^{2}\right.$ surface area) permitted by the Home Office license, the mouse was killed, and the tumour was excised. 


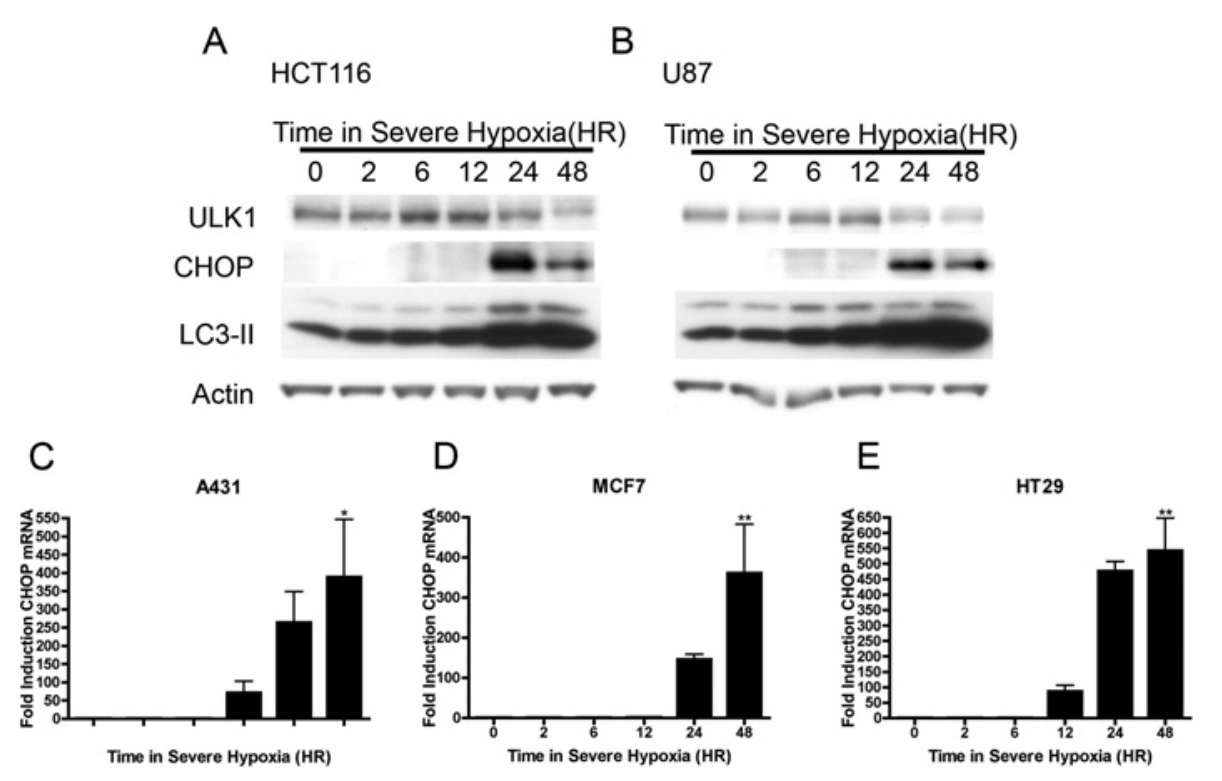

\section{Figure S1 ULK1 and CHOP are induced by severe hypoxia}

HCT116 (A) and U87 (B) cells were exposed to severe hypoxia $\left(<0.01 \% \mathrm{O}_{2}\right)$ for the times indicated after which cells were harvested and analysed by Western blotting using the antibodies indicated. A431 (C), MCF7 (D) and HT29 (E) cells were exposed to severe hypoxia for the times indicated after which cells were harvested and analysed RT-qPCR for CHOP mRNA. 
A

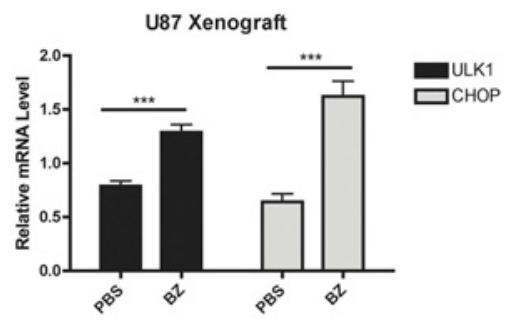

B

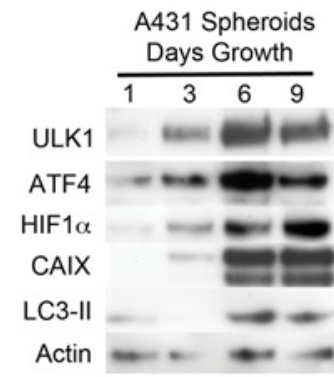

C

A431 Spheroids

PIMO

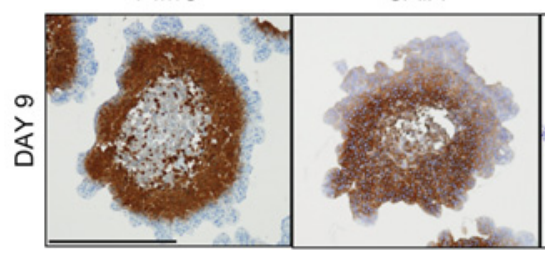

CHOP

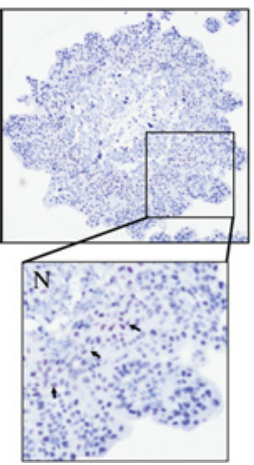

Figure S2 ULK1 is induced in hypoxic xenografts and spheroids

(A) U87 cells were subcutaneously injected into the flanks of SCID (severe combined immunodeficiency) mice, and treated with bevacizumab (BZ; $10 \mathrm{mg} / \mathrm{kg}$ of body weight) or PBS every 3 days from the day of implantation. After tumours reached end point, mice were killed, tumours were harvested and mRNA was extracted for analysis with the primers indicated. (B and C) A431 cells were seeded in round-bottomed 96-well plates at 5000 cells/well overnight. After $24 \mathrm{~h}$ spheroid-like structures formed and began to grow (Day 1). Spheroids were collected on the days indicated and analysed by Western blotting (B) or immunohistochemistry (C) using the antibodies indicated. PIMO, pimonidazole.

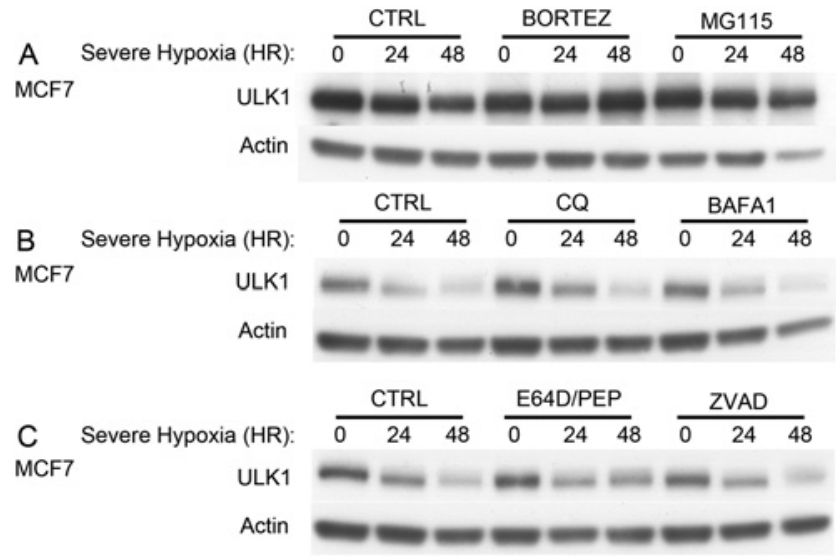

Figure S3 ULK1 degradation in hypoxic MCF7 cells is mediated by the proteasome

MCF7 cells were treated with (A) $100 \mathrm{nM}$ bortezomib (BORTEZ) or $5 \mu \mathrm{M}$ MG115, (B) $25 \mu \mathrm{M}$ chloroquine (CQ) or $0.1 \mu \mathrm{M}$ bafilomycin A1 (BAFA1), or (C) $10 \mu \mathrm{g} / \mathrm{ml}$ of each E64D and pepstatin $\mathrm{A}$ (PEP), or $50 \mu \mathrm{M}$ ZVAD-FMK (ZVAD) and then exposed to severe hypoxia for the times indicated. Cells were harvested and analysed by Western blotting using the antibodies indicated. 
A

A431

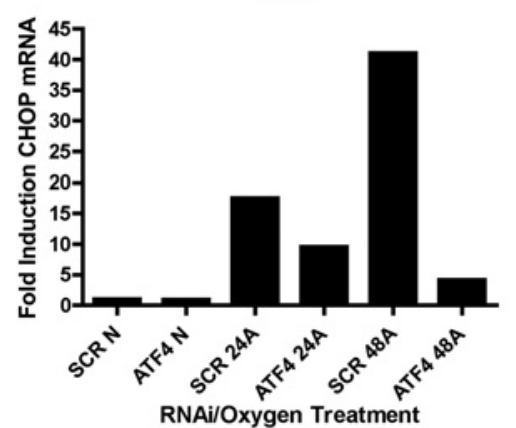

B

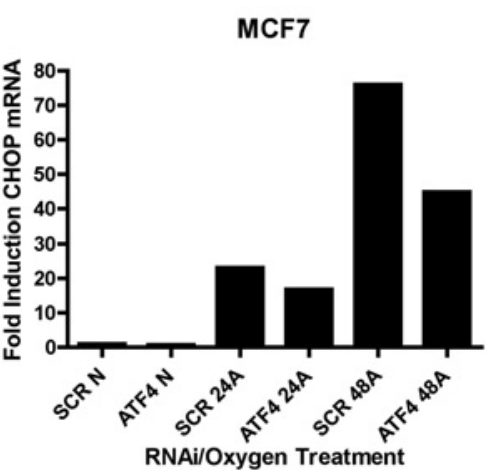

Figure S4 Knockdown of ATF4 reduces CHOP mRNA expression in severe hypoxia

A431 (A) or MCF7 (B) cells were transfected with siRNA against ATF4 or a scrambled control sequence (SCR) overnight and exposed to severe hypoxia (A) or normoxia (N) the following day. After 24 or $48 \mathrm{~h}$, cells were harvested for analysis by RT-qPCR for CHOP mRNA. $n=1$.

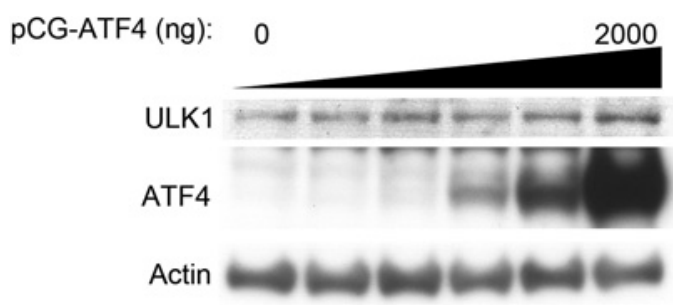

Figure S5 Ectopic expression of ATF4 is insufficient to drive ULK1 protein expression

A431 cells were transfected with 0-2000 ng of pCG-empty vector or pCG-ATF4. At $48 \mathrm{~h}$ later, cells were harvested and analysed by Western blotting using the antibodies indicated. 
A

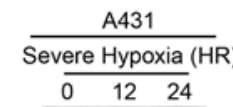

Phospho-MBP

Total MBP (Coomassie)

ULK1 (Immunoprecipitation)

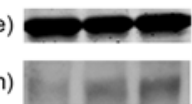

B

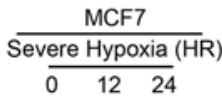

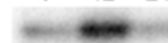

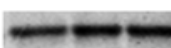

$\mathrm{man}$

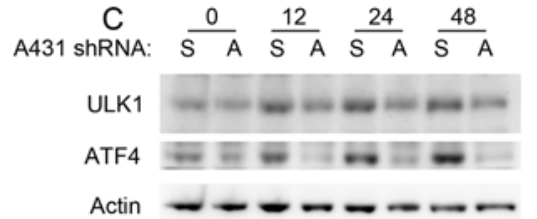

D

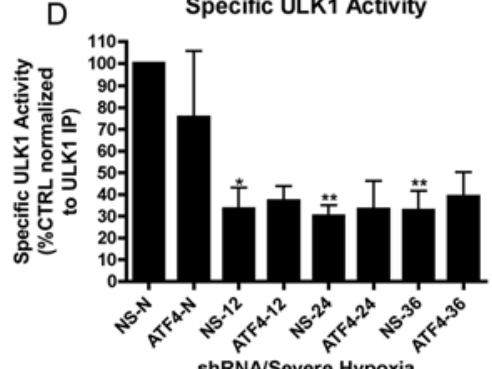

E

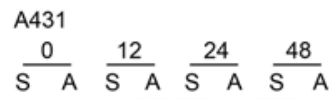

Phospho-MBP

Total MBP (Coomassie)

ULK1 (Immunoprecipitation)

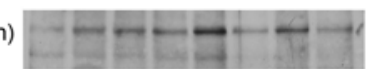

Figure S6 Severe hypoxia increases ULK1 kinase activity in an ATF4dependent manner

A431 cells were exposed to severe hypoxia for 12 or $24 \mathrm{~h}$, after which time cells were harvested and lysates prepared. An antibody specific to ULK1 was used to immunoprecipitate ULK1, and detection of ULK1 kinase activity was performed using MBP as a substrate. Images of P-MBP, Coomassie Blue staining of total MBP and immunoprecipitation of endogenous ULK1 from A431 (A) and MCF7 cells (B) exposed to severe hypoxia are shown. Alternatively, A431 cells expressing an inducible shRNA construct against ATF4 (A) or a non-silencing sequence (S) were treated with doxycycline for 2 days and then exposed to severe hypoxia for the times indicated. Western blots are shown in (C), confirming depletion of ATF4 and the consequent abrogation in ULK1 expression. ULK1 specific activity (normalized to ULK1 protein level) is shown in (D), and P-MBP, Coomassie Blue staining and ULK1 immunoprecipitation are shown in $(\mathbf{E})$.
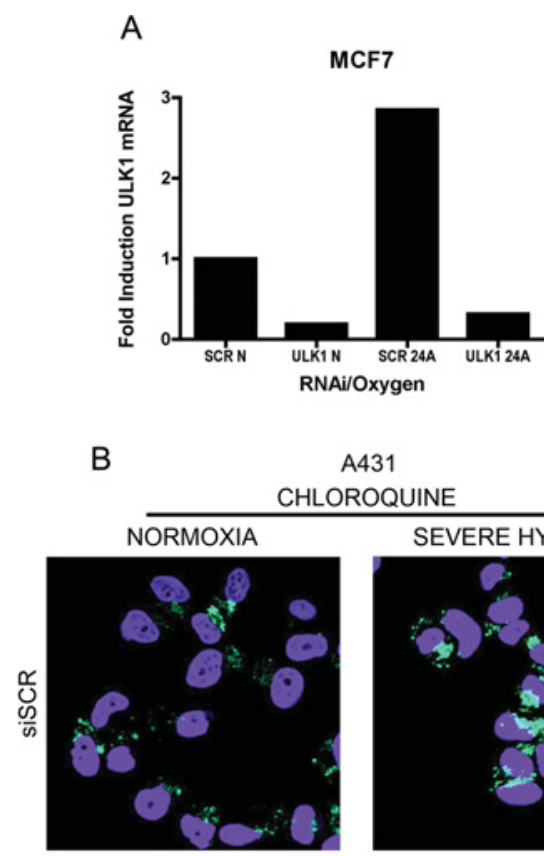

A431
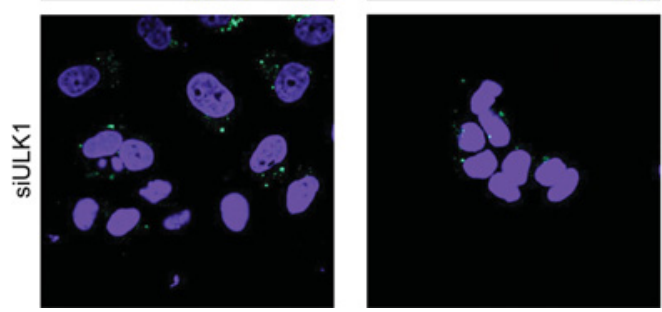

Figure S7 RNAi silencing of ULK1 mRNA reduces autophagic flux

(A) RNAi silencing of ULK1 mRNA. MCF7 cells were transfected with siRNA against ULK1 or a scrambled control sequence (SCR) overnight and exposed to severe hypoxia (A) or normoxia (N) the following day. After 24 or $48 \mathrm{~h}$, cells were harvested for analysis by RT-qPCR for ULK1 mRNA. $n=1$. (B) ULK1 is required for autophagy induction in severe hypoxia. MCF7 cells were transfected with siRNA against ULK1 or a scrambled control sequence (SCR) overnight and reseeded on to coverslips the next day. Cells were treated with $25 \mu \mathrm{M}$ chloroquine and exposed to severe hypoxia for $24 \mathrm{~h}$ and then fixed and stained for LC3 (green) and counterstained with DAPI (4',6-diamidino-2-phenylindole) (blue). 


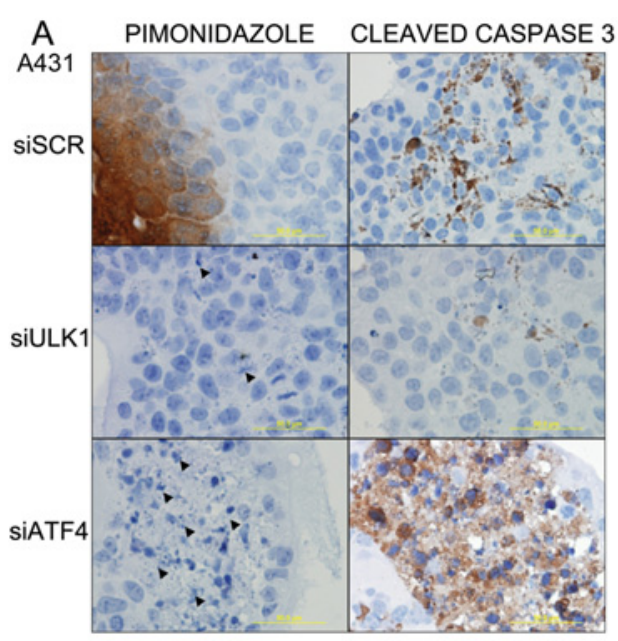

B Spheroid Growth

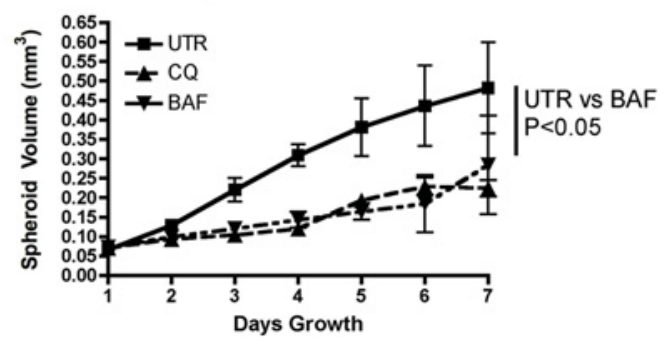

Figure S8 ULK1 protects cancer cells from caspase-3/7-independent cell death

(A) A431 spheroids lacking ULK1 or ATF4, or controls were harvested on day 3 and stained using the antibodies indicated and counterstained with haematoxylin. Arrows point to visible nuclear fragmentation. (B) A431 cells were seeded at 5000 cells/well to a round-bottomed 96-well plate with Matrigel ${ }^{\top \mathrm{M}}$. After $24 \mathrm{~h}$, spheroid-like structures formed and began to grow (Day 1). A single dose of $25 \mu \mathrm{M}$ chloroquine (CQ) or $0.1 \mu \mathrm{M}$ bafilomycin $\mathrm{A} 1$ (BAF) was administered to spheroids on day 1. Images were taken daily and volume measurements were calculated with ImageJ (NIH). $n=10$ spheroids in each group. Regression analysis was applied to calculate the slope of each curve and $P$ values were calculated by one-way ANOVA. UTR, untreated.

\section{REFERENCE}

1 Higgins, G. S., Harris, A. L., Prevo, R., Helleday, T., McKenna, W. G. and Buffa, F. M. (2010) Overexpression of POLQ confers a poor prognosis in early breast cancer patients. Oncotarget 1, 175-184

Received 14 June 2012/25 September 2012; accepted 18 October 2012

Published as BJ Immediate Publication 18 October 2012, doi:10.1042/BJ20120972 
Copyright of Biochemical Journal is the property of Portland Press Ltd. and its content may not be copied or emailed to multiple sites or posted to a listserv without the copyright holder's express written permission. However, users may print, download, or email articles for individual use. 